\title{
O BANCO DE DADOS DO CRITT PARA A PESQUISA DO PROCESSO TRADUTÓRIO ${ }^{1}$
}

THE CRITT TRANSLATION PROCESS RESEARCH DATABASE

\author{
Michael CARL ${ }^{2}$ \\ Moritz SCHAEFFER ${ }^{3}$ \\ Srinivas BANGALORE ${ }^{4}$ \\ Tradução de: Leonardo Penha MESQUITA e Leonardo Lima Beschizza dos SANTOS 5
}

\begin{abstract}
Resumo: Desde sua criação, há dez anos, o Center for Research and Innovation in Translation and Translation Technology (CRITT), da Copenhagen Business School (CBS), Dinamarca, está envolvido em pesquisas do processo tradutório. Os dados dessas pesquisas foram coletados inicialmente pela ferramenta Translog e publicados em 2012 como um banco de dados das pesquisas do processo tradutório (em inglês, Translation Process Research Database, ou TPR-DB). Desde 2012, outros experimentos foram realizados, e mais dados foram adicionados ao TPR-DB. Em particular, dentro do projeto CASMACAT - do inglês, Cognitive Analysis and Statistical Methods for Advanced Computer Aided Translation (SANCHIS-TRILLES et al., 2014) -, foi gravada uma grande quantidade de dados do processo de pós-edição de tradução automática, e o TPR-DB foi disponibilizado sob uma licença Creative Commons. No momento da redação deste artigo, o TPR-DB contém quase 30 estudos com tarefas de tradução, pós-edição, escrita (autoral) e/ou cópia, todas gravadas com o Translog e/ou com a ferramenta CASMACAT. Cada estudo é constituído por cerca de oito a mais de 100 sessões de gravação, envolvendo mais de 300 tradutores. Atualmente, os dados somam mais de 500 horas de produção textual reunidas em mais de 1.400 sessões, com mais de 600.000 palavras traduzidas para mais de dez línguas-alvo diferentes. Este artigo descreve os recursos e as opções de visualização do TPR-DB. Esse banco de dados contém registros do processo, bem como informações derivadas e anotadas organizadas em sete tipos de unidades simples e compostas do processo e do produto, adequadas para investigar os processos de tradução humana e assistida por computador, bem como para realizar modelagem avançada dos usuários.
\end{abstract}

Palavras-chave: pesquisa empírica do processo tradutório, banco de dados de pesquisas do processo tradutório.

\footnotetext{
${ }^{1}$ Agradecemos a Michael Carl e à editora Springer a gentileza de cederem os direitos para a publicação desta versão traduzida. Referência do artigo original: CARL, Michael; SCHAEFFER, Moritz; BANGALORE, Srinivas. The CRITT translation process research database. In: New directions in empirical translation process research: exploring the CRITT TPR-DB. Nova York: Springer, 2015. p. 13-56.

${ }^{2}$ Center for Research and Innovation in Translation and Translation Technology (CRITT), Departamento de Comunicação em Negócios Internacionais, Copenhagen Business School (CBS), Frederiksberg, Dinamarca.

${ }^{3}$ Center for Research and Innovation in Translation and Translation Technology, Departamento de Comunicação em Negócios Internacionais, Copenhagen Business School (CBS), Frederiksberg, Dinamarca.

Instituto de Linguagem, Cognição e Computação, Universidade de Edimburgo, Edimburgo, Reino Unido.

${ }^{4}$ Interactions Corporation, New Providence, NJ, Estados Unidos.

${ }^{5}$ Leonardo Penha Mesquita: Tradutor de inglês e analista de TI. Possui Bacharelado em Tradução pela Universidade Federal de Uberlândia (2015) e experiência no mercado de tecnologia da informação desde 2003. Atua como tradutor de inglês para o portal web <www.wikihow.com> desde 2014 e como analista de TI na LGTi Tecnologia desde 2016. E- mail: lpmesquita@gmail.com. Leonardo Lima Beschizza dos Santos: Estudante do curso de Bacharelado em Tradução na Universidade Federal de Uberlândia (UFU) desde 2016. Email: leonardobeschizza@live.com. Tradução supervisionada pelo Prof. Dr. Igor Antonio Lourenço da Silva.
} 


\section{Introdução}

A pesquisa empírica do processo tradutório demanda a disponibilidade de dados adequados do processo. A fim de permitir uma pesquisa empiricamente fundamentada do processo de tradução, Jakobsen e Schou (1999) idealizaram, em 1995, uma ferramenta para registrar os acionamentos de teclas e mouse (key logging), o Translog, que permite a gravação de sessões de tradução, a visualização dos dados e a realização de análises estatísticas. Desde então, o Translog foi submetido a inúmeras mudanças, tanto no que diz respeito à aquisição de dados quanto no que tange ao formato e à representação dos processos coletados (cf. JAKOBSEN 2011, traduzido neste volume), para permitir uma análise mais robusta dos dados. O Translog-II (CARL, 2012a) atual foi complementado com a interface do CASMACAT - do inglês, Cognitive Analysis and Statistical Methods for Advanced Computer Aided Translation (SANCHIS-TRILLES et al., 2014) -, uma ferramenta de pósedição de tradução automática utilizando um navegador; e os dados brutos obtidos ao final das sessões gravadas de tradução podem ser complementados com anotações e convertidos em um banco de dados de pesquisas do processo tradutório (em inglês, Translation Process Research Database, ou TPR-DB). Até o momento, o TPR-DB acumulou uma grande quantidade de dados do processo, com o objetivo de:

1. representar, de forma consistente, dados de atividade obtidos na pesquisa do processo tradutório de modo a viabilizar a investigação a partir de centenas de sessões de tradução, considerando línguas diferentes e modos distintos de tradução; e

2. implementar e disponibilizar um grande número de recursos para todo o conjunto de dados coletados, algo que seria difícil ou praticamente impossível de se calcular individualmente para cada sessão.

Dessa forma, o objetivo do TPR-DB é estimular a investigação e reduzir as barreiras de entrada para a pesquisa, em grande escala, do processo tradutório, facilitada por um formato consistente de banco de dados e um conjunto de recursos bem definidos. ${ }^{6}$

O TPR-DB é organizado em estudos e sessões. Um estudo é uma coleção de sessões conduzidas no mesmo contexto experimental. O Translog e o CASMACAT geram um único arquivo de registro para cada sessão. Em seguida, esses dados brutos do registro são anotados e processados em um conjunto em tabelas que contêm ampla gama de recursos e atributos.

\footnotetext{
${ }^{6} \mathrm{O}$ banco de dados está disponível gratuitamente sob uma licença Creative Commons e pode ser baixado em: $<\mathrm{https}$ ://sites.google.com/site/centretranslationinnovation/tpr-db $>$.
} 
Este artigo descreve as tabelas e os recursos extraídos dos dados registrados e anotados. A Seção 2 fornece uma visão geral do TPR-DB; descreve o processo de anotação dos dados registrados em uma sessão de tradução, explica seu mapeamento para o TPR-DB e fornece uma visão geral das tabelas do TPR-DB. As Seções 3 a 5 descrevem as tabelas mais detalhadamente: a Seção 3 apresenta as tabelas que codificam os acionamentos de teclas e de mouse e as fixações; a Seção 4 ilustra as tabelas das unidades de produção e unidades de fixação, unidades essas que configuram como propriedade especial a sinalização de comportamentos de leitura e digitação paralelas e/ou alternadas, os quais apontam a carga de trabalho cognitivo de um tradutor; a Seção 5 expõe as tabelas das unidades do produto tradutório, ou seja, as unidades derivadas do produto final da tradução: itens-fonte (source tokens), itens-alvo (target tokens) e unidades de alinhamento (alignment units). A Seção 6 mostra as possibilidades de visualização dos dados do processo. A Seção 7 sugere as possibilidades de adição de recursos gerados externamente ao TPR-DB. Por fim, três apêndices complementam este artigo: os Apêndices 1 e 2 fornecem uma visão geral dos estudos no TPR-DB; o Apêndice 3 apresenta uma lista completa dos recursos disponíveis.

\section{Visão geral do TPR-DB}

O banco de dados disponibilizado ao público geral pelo CRITT (Center for Research and Innovation in Translation and Translation Technology, Copenhagen Business School, Copenhagen, Dinamarca) reúne seções gravadas de tradução (e de outras formas de produção textual) para fins de pesquisa do processo tradutório. Contém dados de atividade do usuário (do inglês, user activity data, ou UAD) referentes aos comportamentos de tradutores coletados em quase 30 estudos envolvendo tarefas de tradução, pós-edição, revisão, escrita (autoral) e/ou cópia, todas gravadas com a interface CASMACAT (SANCHIS-TRILLES et al., 2014) e/ou o software Translog-II (CARL, 2012a). Cada estudo é constituído por cerca de oito a mais de 100 sessões de gravação. Atualmente, o banco de dados soma mais de 500 horas de produção textual reunidas em mais de 1.400 sessões, contendo, ao todo, mais de 600.000 palavras traduzidas para mais de dez línguas-alvo. O website do TPR-DB disponibiliza, sob uma licença Creative Commons, todos os dados registrados durante os processos tradutórios ( $>20 \mathrm{~GB})$, bem como uma série de anotações adicionais aos conjuntos de sessões (arquivo de 170 MB compactado em formato .zip). Nesta seção, descreve-se como os dados registrados durante uma sessão de tradução são inseridos no TPR-DB. 


\subsection{Compilação do TPR-DB}

Os dados brutos de atividade do usuário (UAD) - que incluem os dados do processo tradutório (e.g., fixações e acionamentos de teclas e mouse) e os dados do produto tradutório (i.e., o texto-fonte e o produto final da tradução) - são armazenados e mantidos em um repositório de especifico. Dentro do processo de compilação do TPR-DB ${ }^{7}$ (CARL, 2012b), diversas tabelas são geradas a partir dos UAD brutos, podendo então ser utilizadas como base para análises e visualizações mais detalhadas.

A Figura 1 mostra um fluxograma do processo de compilação do TPR-DB. Os UAD registrados (rotulados como Translog-II na figura) são processados em dois fluxos independentes para anotar os dados do produto (parte superior) e os dados do processo (parte inferior).

Figura 1 - Arquitetura do processo de compilação do TPR-DB

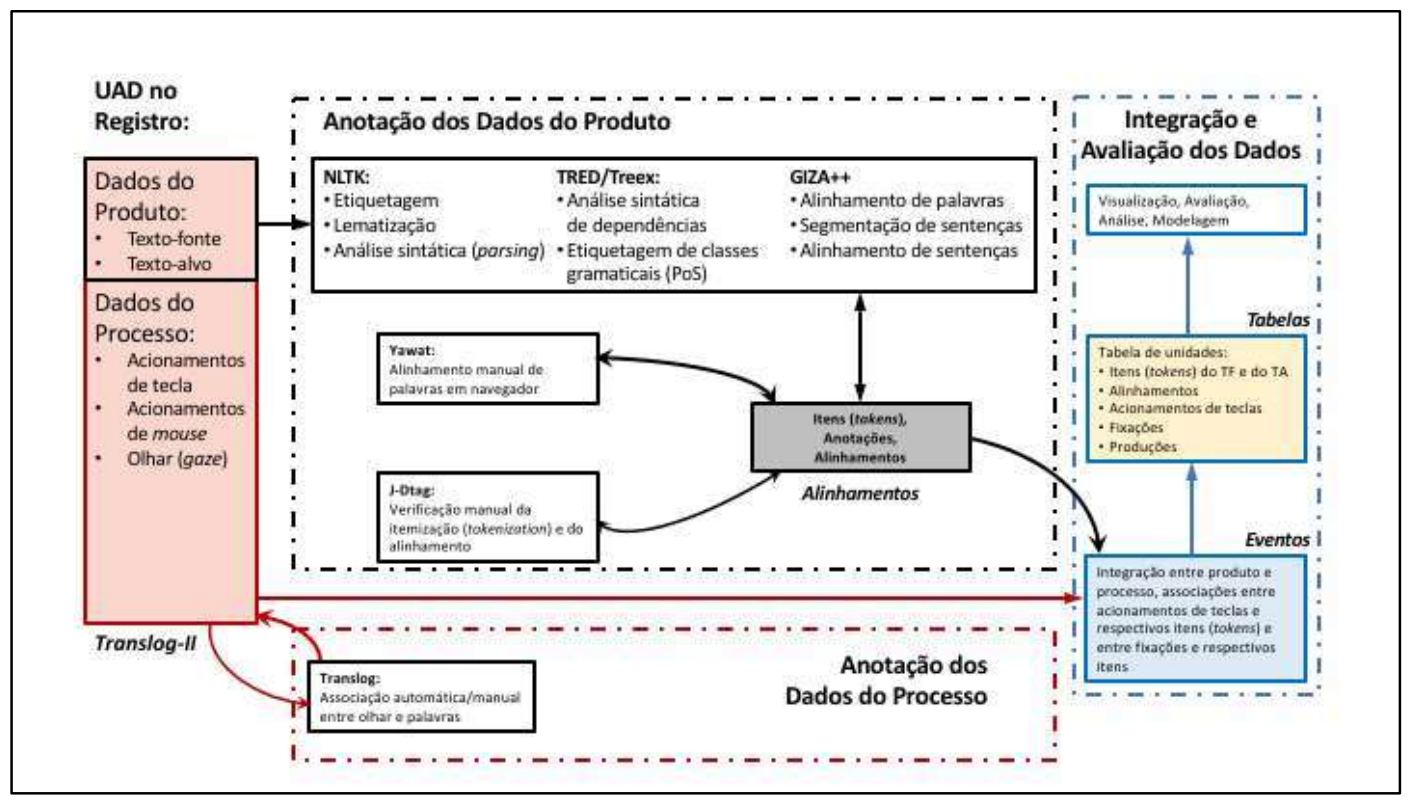

As anotações dos dados do produto, isto é, dos textos-fonte e do texto-alvo, incluem a itemização (tokenization), o alinhamento das sentenças e das palavras (tokens), bem como, de forma opcional, a lematização e a etiquetagem de classes gramaticais ( $\mathrm{PoS}$ - do inglês, parts of speech), dentre outros processos. O Translog-II também oferece a possibilidade de ajustar e anotar os dados do processo, como o remapeamento das associações entre os dados do olhar e as respectivas palavras fixadas. Em seguida, uma etapa de integração dos dados registra as

\footnotetext{
${ }^{7}$ Embora os registros dos UAD sejam um pouco distintos no Translog-II e no CASMACAT, a estrutura das tabelas geradas é idêntica.
} 
associações dos acionamentos de teclas e das fixações às respectivas palavras de que fazem parte (cf. CARL, 2012a). Por fim, são produzidas diversas tabelas diferentes, as quais disponibilizam um grande número de recursos e descrevem vários tipos de unidades de produto e de processo, conforme descrito na Seção 2.3.

O processo de compilação do TPR-DB é completamente automático, mas fornece uma interface gráfica do usuário (do inglês, graphical user interface, ou GUI), na qual o alinhamento das palavras pode ser ajustado para semiautomático. A Figura 2 mostra a interface gráfica do usuário do alinhador YAWAT (GERMANN, 2008), na qual os alinhamentos por palavra podem ser realçados, verificados e alterados.

Figura 2 - Capturas de tela do YAWAT, ferramenta de alinhamento de palavras que roda na interface de um navegador

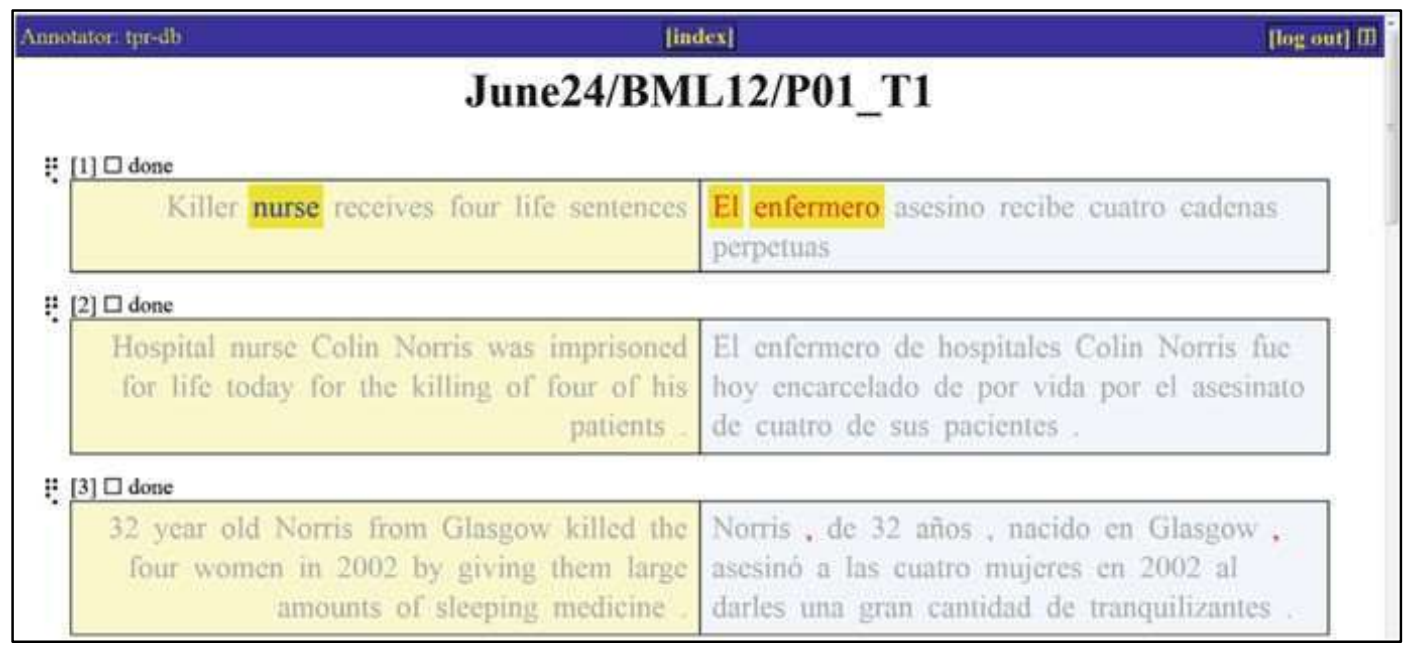

\subsection{Estudos do TPR-DB}

Cada estudo é uma coleção coerente de sessões de tradução ou de produção textual, podendo envolver diferentes tarefas. Todos os estudos do TPR-DB contêm um registro dos acionamentos de teclas e mouse, e uma grande quantidade deles também contém dados de rastreamento ocular (eye tracking). Cada estudo do TPR-DB foi conduzido com (um conjunto de) pergunta(s) de pesquisa em mente, mas os estudos do banco de dados podem ser brevemente resumidos da seguinte forma:

- nove estudos foram conduzidos com as diferentes interfaces do CASMACAT para testar e avaliar suas diferentes funcionalidades;

- sete estudos são parte de um experimento multilíngue que visou comparar tradução do zero, pós-edição bilíngue e pós-edição monolíngue feitas do inglês 
para uma de seis línguas-alvo por mais de 120 tradutores diferentes.

- alguns experimentos individuais foram realizados com o Translog-II com os propósitos descritos no Apêndice 1.

O Apêndice 1 fornece uma visão geral detalhada sobre diversos estudos reunidos no TPR-DB, bem como seus propósitos, participantes e durações. A Tabela 1 é um excerto do Apêndice 1 e mostra uma informação resumida do estudo CFT $14 .^{8}$

Tabela 1 - Excerto da tabela do Apêndice 1

\begin{tabular}{c|c|c|c|c|c|c|c|c|c|c|c}
\hline Study & Task & Part & Sess & Texts & SL & TL & FDur & KDur & PDur & SLen & TLen \\
\hline CFT14 & R & 4 & 14 & 14 & en & es & 4.54 & 1.21 & 0.28 & 2901 & 40,614 \\
\hline CFT14 & P & 7 & 7 & 2 & en & es & 16.51 & 7.90 & 3.41 & 2901 & 20,273 \\
\hline CFT14 & PIO & 7 & 7 & 2 & en & es & 15.68 & 7.98 & 3.49 & 2901 & 20,341 \\
\hline
\end{tabular}

Cada estudo é constituído por uma ou mais sessões (Sess) de gravação, com diferentes números de participantes (Part), diferentes textos, diferentes tarefas e diferentes direções da tradução, considerando a língua-fonte (SL) e a língua-alvo (TL). Por exemplo, o estudo CFT14 na Tabela 1 possui três tarefas distintas (R, P e PIO) ${ }^{9}$, nas quais dois textos-fonte em inglês $(\mathrm{SL}=\mathrm{en})$ foram pós-editados em espanhol $(\mathrm{TL}=\mathrm{es})$. Além disso, sete participantes produziram sete traduções (sessões) para cada P e para cada POI; e quatro participantes revisaram posteriormente os 14 textos pós-editados.

O tempo total de produção é indicado por FDur, KDur e PDur, siglas que representam a soma das durações de todas as sessões, menos as pausas encontradas antes do acionamento da primeira tecla e após o acionamento da última tecla, bem como as pausas entre os acionamentos de teclas consecutivos (dependendo da duração da pausa):

- Dur representa a duração total da sessão;

- FDur exclui as pausas maiores que $200 \mathrm{~s}$ entre os acionamentos de teclas e mouse consecutivos;

- KDur é o tempo da sessão menos as pausas maiores que $5 \mathrm{~s}$ entre os acionamentos de teclas e mouse; e

- PDur indica o tempo de digitação sem pausas maiores que $1 \mathrm{~s}$ entre os

\footnotetext{
${ }^{8} \mathrm{NT}$ : Observe-se que os termos e os algarismos, nesta e em outras tabelas, são dispostos conforme o original em língua inglesa, para manter a forma como se visualizam as informações no banco de dados.

${ }^{9} \mathrm{O}$ exemplo foi tirado de um estudo utilizando o CASMACT. As tarefas são: R (revisão), P (pós-edição) e POI (pós-edição interativa com aprendizado online). Há uma lista com as descrições completas de cada uma delas no Apêndice 1.
} 
acionamentos de teclas e mouse.

$\mathrm{Na}$ Tabela 1, a duração total (FDur) das pós-edições (P) dos sete textos pelos sete póseditores foi de 16,51 h. Dois atributos adicionais de duração indicam as durações da digitação. De acordo com o valor de KDur, os pós-editores digitaram cerca de $50 \%$ do tempo total $(7,90 \mathrm{~h})$, ao passo que, com base no valor de PDur, esse tempo foi de apenas 3,41 h, ou aproximadamente $20 \%$ do tempo total de pós-edição. ${ }^{10}$ A Tabela 1 também mostra o comprimento médio do texto-fonte (SLen) e o número total de palavras produzidas na línguaalvo (TLen).

\subsection{Tabelas de Resumo do TPR-DB}

O processo de compilação do TPR-DB projeta os UAD brutos em diferentes unidades de produto e de processo que são reunidas nas tabelas do banco de dados. Cada linha de uma tabela descreve uma unidade particular com diversos atributos que serão descritos detalhadamente nas Seções 3 a $5^{11}$ :

As tabelas com as unidades de produto básicas são:

1. itens-fonte (do inglês, source tokens, ou ST): essa tabela lista os itens (tokens) do texto-fonte, junto com seus correspondentes no texto-alvo, o número de inserções e exclusões necessárias para produzir a tradução, informações das microunidades etc. (Seção 4.6);

2. itens-alvo (do inglês, target tokens, ou TT): essa tabela lista os itens do texto-alvo, junto com seus correspondentes no texto-fonte, número de inserções e exclusões necessárias para produzir o texto-alvo, informações da microunidade, quantidade de atividade de leitura paralela à digitação etc. (Seção 4.6).

As tabelas com as unidades de produto compostas são:

3. sessão (do inglês, session, ou SS): essa tabela descreve as propriedades relacionadas à sessão, como língua-fonte, língua-alvo, duração total da sessão, início e final da redação (drafting) (Seção 3.1);

\footnotetext{
10 Diferentes valores para delimitação de pausas já foram sugeridos e utilizados. Vandepitte et al. (2015) segmentam as sequências de acionamentos de teclas considerando 200 ms, enquanto Lacruz e Shreve (2014, p. 250) sugerem que "eventos completos de edição são separados por pausas longas (de $5 \mathrm{~s}$ ou mais). Eles geralmente contêm pausas curtas (superiores a $0,5 \mathrm{~s}$, mas inferiores a $2 \mathrm{~s}$ ), e os eventos completos de edição que demandam mais esforço geralmente incluem múltiplas pausas curtas. Os pós-editores podem fazer pausas de duração intermediária (superiores a $2 \mathrm{~s}$, mas inferiores a $5 \mathrm{~s}$ ) durante um evento completo de edição". Jakobsen (2005) sugere 2,4 s para a sua definição de "desempenho de pico" em tradução.

${ }^{11}$ As letras entre parênteses na lista representam a extensão do arquivo no TPR-DB. As seções indicam onde a tabela é descrita com maiores detalhes.
} 
4. segmentos (do inglês, segments, ou SG): essa tabela lista as propriedades dos segmentos alinhados entre o texto-fonte e o texto-alvo, dentre as quais estão o número de inserções e exclusões, bem como o número de duração das fixações (Seção 3.2);

5. unidades de alinhamento (do inglês, alignment units, ou AU): essa tabela lista as unidades de alinhamento entre o texto-fonte e o texto-alvo, junto com o número acionamentos de teclas e mouse (inserções e exclusões) necessários para produzir a tradução, informações sobre as microunidades, quantidade de atividades de leitura paralela à produção das AU etc. (Seção 4.1).

As tabelas com as unidades de processo básicas são:

6. dados dos acionamentos de teclas (do inglês, keystroke data, ou KD): essa tabela enumera as operações de modificação do texto (inserções e exclusões), junto com o tempo dos acionamentos de teclas e mouse, bem como a palavra no texto final para a qual o acionamento de tecla contribui (Seção 5.1);

7. dados de fixação (do inglês, fixation data, ou FD): essa tabela enumera as fixações no texto-fonte ou no texto-alvo definidas pelo tempo inicial, tempo final e sua respectiva duração, bem como a posição do caractere e da palavra fixada na janela do texto-fonte ou do texto-alvo (Seção 5.2).

As tabelas com as unidades de processo compostas são:

8. unidades de produção (do inglês, production units, ou PU): essa tabela lista as unidades de sequência contínua de atividade de digitação da sessão, definidas pelo tempo inicial, tempo final e sua respectiva duração, porcentagem de atividade de leitura paralela à produção da unidade, duração da pausa na produção antes do início da digitação, bem como o número de inserções e exclusões (Seção 5.3);

9. unidades de fixação (do inglês, fixation units, ou FU): essa tabela lista as sequências contínuas de atividade de leitura, caracterizadas pelo tempo inicial, tempo final e sua respectiva duração, bem como os índices do percurso do olhar pelas palavras fixadas (Seção 5.4);

10. unidades de atividade (do inglês, activity units, ou CU): essa tabela fornece uma lista dos fragmentos da sessão, sendo cada fragmento definido por atividades de digitação ou leitura do texto-fonte ou do texto-alvo (Seção 5.5).

Além disso, o uso de fontes externas está resumido em:

11. fontes externas (do inglês, external resources, ou EX): essa tabela lista os dados 
de acionamentos de teclas e mouse registrados com o Inputlog (LEITEN; WAES 2013) (Seção 7.1).

\section{Informações do resumo das sessões e dos segmentos}

Dependendo do desenho, um estudo compreende uma ou mais sessões. Durante uma sessão, um texto é traduzido, copiado, editado ou revisado, sendo que cada texto possui diversas sentenças (segmentos). Esta seção descreve as informações do resumo das sessões e dos segmentos.

\subsection{Informações do resumo das sessões}

As informações do resumo da sessão estão contidas na tabela "sessão" (SS), conforme consta nas Tabelas 2 a 4. Essas tabelas apresentam as informações conforme descrito a seguir.

a. As informações gerais da sessão incluem:

- o nome do estudo (Study) e da sessão (Session), ou seja, o nome do diretório e do arquivo de registro;

- a língua-fonte e a língua-alvo (SL e TL, respectivamente);

- um identificador dos participantes exclusivo para o estudo (Part);

- um identificador do texto exclusivo para o estudo (Text);

- o tipo da tarefa (Task) (conforme apresentado na Tabela 1);

- um identificador do segmento exclusivo para o estudo, tanto no texto-fonte (SegST) quanto no texto-alvo (SegTT), conforme discutido abaixo;

- TokS e TokT indicam, respectivamente, o número de itens (tokens) no textofonte e no texto-alvo;

- LenS e LenT indicam, respectivamente, o comprimento, em caracteres, do texto-fonte e o comprimento do texto-alvo.

b. As informações sobre as durações da sessão indicam quanto tempo levou o processamento dos seguintes itens:

- a duração total da sessão (Dur) e a duração da pausa (Pause) caso hajam ocorrido interrupções na sessão;

- o tempo de início da redação (drafting) (TimeD) e o tempo de início da revisão (TimeR). TimeD indica o tempo transcorrido desde o início da sessão até o primeiro toque do teclado, coincidindo com o final da fase de orientação. 
TempoR indica o tempo em que a fase de redação terminou, dando início à fase da revisão; seu início coincide com o final da primeira microunidade (cf. a seguir) em que é traduzida a última palavra do texto-fonte (cf. JAKOBSEN, 2002, traduzido neste volume);

- as durações FDur, KDur e PDur já foram apresentadas anteriormente. O intervalo PDur fragmenta os UAD em unidades de produção (PU), que serão discutidas na Seção 5. Pnum representa o número de PU em uma sessão.

c. As informações sobre o processamento da sessão indicam os acionamentos de teclas e mouse e o comportamento do olhar:

- FixF e FixD são, respectivamente, os números de fixações no(s) iten(s)-fonte e no(s) iten(s)-alvo, enquanto TrtS e TrtT representam o tempo total de leitura, ou seja, a soma das durações de todas as fixações no texto-fonte e texto-alvo, respectivamente;

- Mins e Mdel representam, respectivamente, os números de caracteres inseridos e excluídos de forma manual, enquanto Ains e Adel representam, respectivamente, os números de caracteres inseridos e excluídos de forma automática. Ains e Adel dizem respeito à pós-edição no CASMACAT, onde o texto editado pode ser programaticamente alterado no modo de interatividade;

- Scatter indica a frequência com a qual a digitação não ocorreu em sequência, isto é, com que frequência o tradutor ou editor sucessivamente digitou teclas que foram parte de duas ou mais palavras diferentes.

Tabela 2 - Informações gerais da sessão, incluindo comprimento do texto-fonte e texto-alvo em termos de palavras e caracteres

\begin{tabular}{c|c|c|c|c|c|c|c|c|c|c|c|c|c}
\hline Study & Session & SL & TL & Part & Text & Task & SegST & SegTT & TokS & LenS & TokT & LenT & $\ldots$ \\
\hline BML12 & P01_E5 & en & es & P01 & 5 & E & 6 & 6 & 139 & 788 & 153 & 840 & $\ldots$ \\
\hline BML12 & P01_P4 & en & es & P01 & 4 & P & 5 & 5 & 110 & 668 & 131 & 763 & $\ldots$ \\
\hline BML12 & P01_T1 & en & es & P01 & 1 & T & 10 & 10 & 160 & 838 & 180 & 964 & $\ldots$ \\
\hline
\end{tabular}

Tabela 3 - Informações sobre as durações da sessão

\begin{tabular}{c|c|c|c|c|c|c|c|c|c}
\hline$\ldots$ & Dur & TimeD & TimeR & Pause & Fdur & Kdur & Pdur & Pnum & $\ldots$ \\
\hline$\ldots$ & 310.234 & 114.140 & 232.656 & 0 & 167.110 & 80.374 & 23.366 & 29 & $\ldots$ \\
\hline$\ldots$ & 268.328 & 71.234 & 264.765 & 0 & 193.531 & 29.407 & 14.485 & 15 & $\ldots$ \\
\hline$\ldots$ & 757.281 & 92.016 & 290.391 & 0 & 654.812 & 314.378 & 210.415 & 72 & $\ldots$ \\
\hline
\end{tabular}


Tabela 4 - Informações sobre o processamento da sessão no estudo BML12

\begin{tabular}{c|c|c|c|c|c|c|c|c|c}
\hline$\ldots$ & FixS & TrtS & FixT & TrtT & Scatter & Mins & Mdel & Ains & Adel \\
\hline$\ldots$ & 3 & 167 & 661 & 68.214 & 17 & 85 & 93 & 0 & 0 \\
\hline$\ldots$ & 551 & 78.224 & 236 & 18.668 & 9 & 77 & 62 & 0 & 0 \\
\hline$\ldots$ & 1122 & 115.692 & 392 & 26.605 & 30 & 1152 & 186 & 0 & 0 \\
\hline
\end{tabular}

As Tabelas 2 a 4 apresentam três sessões do estudo BML12 conduzidas pelo participante P01. O texto (Text) 5 foi editado $($ Task $=\mathrm{E})$; o texto 4, pós-editado $($ Task $=\mathrm{P})$; e o texto 1 , traduzido do zero $($ Task $=\mathrm{T})$. A tradução levou mais tempo em termos de todas as medidas de duração disponíveis, Dur, FDur, KDur e PDur, enquanto a pós-edição foi mais rápida que a edição em relação aos valores de Dur, KDur e PDur, mas mais lenta em relação ao valor de FDur. Observe-se que a edição foi uma atividade mais fragmentada do que a pósedição, pois foi produzida uma quantidade maior de PUs.

\subsection{Informações do resumo dos segmentos}

O nome da sessão P01_T1 significa que o participante P01 traduziu (T) o texto 1. Os 11 segmentos do texto-fonte (STseg) foram traduzidos em dez segmentos no texto-alvo (TTseg). As propriedades desses segmentos são exibidas com maior detalhamento nas tabelas de resumo dos segmentos (SG), conforme ilustrado na Tabela 5.

Tabela 5. Informações processuais das unidades de alinhamento

\begin{tabular}{c|c|c|c|c|c|c|c|c|c|c|c|c}
\hline STseg & TTseg & Study & Session & Nedit & Dur & $\ldots$ & Scatter & Literal & HTra & HSeg & CrossS & CrossT \\
\hline 1 & 1 & BML12 & P01_T1 & 2 & 20,028 & $\ldots$ & 2 & 27.93 & 2.16 & 1.18 & 2 & 1.29 \\
\hline 2 & 2 & BML12 & P01_T1 & 3 & 38,951 & $\ldots$ & 5 & 48.24 & 1.23 & 0.67 & 2 & 1.29 \\
\hline 3 & 3 & BML12 & P01_T1 & 2 & 83,452 & $\ldots$ & 5 & 67.41 & 1.7 & 0.95 & 1.57 & 1.08 \\
\hline 4 & 4 & BML12 & P01_T1 & 4 & 73,292 & $\ldots$ & 4 & 29.45 & 1.74 & 0.8 & 1 & 1.29 \\
\hline 5 & 5 & BML12 & P01_T1 & 3 & 24,373 & $\ldots$ & 3 & 31.67 & 1.84 & 0.79 & 1.14 & 1.5 \\
\hline 6 & 6 & BML12 & P01_T1 & 2 & 14,030 & $\ldots$ & 2 & 33.3 & 2.43 & 1.36 & 1.3 & 1.09 \\
\hline 7 & 7 & BML12 & P01_T1 & 2 & 58,966 & $\ldots$ & 4 & 19.65 & 0.97 & 0.46 & 1.47 & 0.94 \\
\hline 8 & 8 & BML12 & P01_T1 & 2 & 40,779 & $\ldots$ & 4 & 151.9 & 2.9 & 1.59 & 2.94 & 1.19 \\
\hline 9 & 9 & BML12 & P01_T1 & 1 & 32,812 & $\ldots$ & 1 & 31.6 & 1.38 & 0.72 & 1.21 & 1.1 \\
\hline $10+11$ & 10 & BML12 & P01_T1 & 3 & 61,326 & $\ldots$ & 6 & 29.11 & 1.24 & 0.61 & 1.67 & 1.28 \\
\hline
\end{tabular}


As tabelas de resumo dos segmentos contêm diversas informações parecidas com aquelas das tabelas das sessões, mas cada uma de suas linhas representa um segmento, e não uma sessão. Sendo assim, Dur, FDur, KDur e PDur representam o tempo de tradução do segmento, e não o tempo de tradução da seção, conforme visto na Tabela 5. As colunas STseg e TTseg indicam as informações de alinhamento do segmento (no caso da Tabela 5, todos os segmentos, estão alinhados um a um, à exceção dos dois últimos, dado que os segmentosfonte - sentenças - 10 e 11 foram traduzidos como o segmento-alvo 10).

Nedit indica com que frequência o segmento foi revisado. Um número maior que 1 sinaliza que o tradutor primeiro fez um "rascunho" da tradução para depois retomá-lo e revisá-lo. Por exemplo, o segmento 4 foi esboçado e depois revisado três vezes, ao passo que o STseg 9 foi o único não revisado durante o processo tradutório.

Literal, HTra, HSeg, CrossS e CrossT serão abordados mais detalhadamente nas Seções 4.4 e 4.6. CrossS e CrossT medem a grau de similaridade sintática entre o texto-fonte e o texto-alvo. HTra e HSeg representam, respectivamente, a média de palavras traduzidas e a entropia média de segmentação, enquanto Literal corresponde à soma do produto de HTra e CrossS.

\section{Informações do resumo dos dados no nível da palavra}

Esta seção introduz as unidades de alinhamento (AU) de nível inferior (baseada em palavras), os itens (tokens) do texto-fonte (ST) e os itens do texto-alvo (TT). Como a maioria dos atributos das AU também ocorre nas unidades do texto-fonte e do texto-alvo, começaremos pela apresentação das AU na Seção 4.1. Na Seção 4.2, discutiremos as representações das microunidades; e, na Seção 4.3, uma métrica de (in)eficiência na digitação. A Seção 4.4 apresenta o atributo Cross, que quantifica as distorções sintáticas entre o textofonte e texto-alvo. Os itens do texto-fonte e do texto-alvo são introduzidos na Seção 4.5, seguidos de informações mais detalhadas sobre o olhar (Seção 4.6) e da entropia na tradução das palavras (Seção 4.7).

\subsection{Unidades de alinhamento}

Os itens-fonte e os itens-alvo correspondem a sequências de caracteres, geralmente separadas por um espaço em branco, enquanto as unidades de alinhamento (AU) são correspondências, de m para $\mathrm{n}$, entre itens-fonte e itens-alvo. As tabelas de unidades fornecem 
informações similares para esses três tipos de unidades. Essas tabelas contêm:

- informações gerais: o nome do estudo (Study) e da sessão (Session), o tipo de tarefa (Task), o identificador do participante (Part), o número do texto (Text), o número do segmento-fonte e dos segmentos-alvo (SFseg e TTseg, respectivamente), a língua-fonte e a língua-alvo;

- informações do produto: as cadeias de caracteres da língua-fonte e da língua-alvo (SAU e TAU, respectivamente), o número de itens (tokens) dessas cadeias (SAUnbr e TAUnbr) e a relação entre os itens do texto-fonte e os do texto-alvo em termos de valores do atributo Cross (cf. Seção 4.5);

- informações do processo: o número acionamentos de teclas (inserções e exclusões), a duração da produção, o comportamento do olhar em termos de números de fixações nas cadeias de caracteres-fonte e alvo da AU (FixS e FixT), o tempo total de leitura (TrtS e TrtT) e a duração da primeira passagem do olhar nas cadeias de caracteres-fonte e alvo (FPDrS e FPDurT). Esses atributos serão explicados na Seção 4.6

Tabela 6 - Informações gerais das unidades de alinhamento

\begin{tabular}{c|c|c|c|c|c|c|c|c|c|c|c|c|c}
\hline AUid & STseg & TTseg & Study & Session & SL & TL & Task & Text & Part & SAU & TAU & SAUnbr & TAUnbr \\
\hline 44 & 3 & 3 & BML12 & P01_T1 & en & es & T & 1 & P01 & of & de & 1 & 1 \\
\hline 45 & 3 & 3 & BML12 & P01_T1 & en & es & T & 1 & P01 & $\begin{array}{c}\text { sleeping_ } \\
\text { medicine }\end{array}$ & $\begin{array}{c}\text { tranquili- } \\
\text { zantes }\end{array}$ & 2 & 1 \\
\hline
\end{tabular}

Tabela 7 - Informações do processo das unidades de alinhamento (parte 1)

\begin{tabular}{c|c|c|c|c|c|c|c|c}
\hline Ins & Del & Dur & FixS & FPDurS & TrtS & FixT & FPDurT & TrtT \\
\hline 24 & 21 & 11,407 & 2 & 167 & 167 & 18 & 50 & 1232 \\
\hline 15 & 0 & 1610 & 27 & 631 & 1896 & 8 & 465 & 615 \\
\hline
\end{tabular}

Tabela 8. Informações do processo das unidades de alinhamento (parte 2)

\begin{tabular}{l|l|l|l}
\hline Cross & InEff & Munit & Edit \\
\hline 1 & 15 & 2 & de_medicinas_para_dormir[rimrod_arap_sanicidem] \\
\hline 2 & 0.94 & 1 & tranquilizantes \\
\hline
\end{tabular}


As Tabelas 6 a 8 exibem a tradução inglês $\rightarrow$ espanhol das $\mathrm{AU}_{44}$ e $\mathrm{AU}_{45}$, relativas respectivamente a "of $\leftrightarrow d e$ " e "sleeping medicine $\leftrightarrow$ tranquilizantes". Conforme indicado nas colunas $\mathrm{SAUnbr}$ e $\mathrm{TAUnbr}, \mathrm{AU}_{44}$ consiste em uma correspondência direta, ao passo que $\mathrm{AU}_{45}$ apresenta uma correspondência de dois para um. A coluna Edit (Tabela 8) traça a sequência de acionamentos de teclas realizados para produzir a tradução. $\mathrm{Na} \mathrm{AU}_{44}$, primeiramente foi digitada a expressão "de medicinas para dormir", que depois teve todos seus itens excluídos à exceção da preposição "de", enquanto, na $\mathrm{AU}_{45}$, a palavra "tranquilizantes" foi digitada sem qualquer revisão. A Tabela 7 mostra os números gerais dos acionamentos de teclas: na $\mathrm{AU}_{44}$, ocorreram 24 inserções, das quais 21 caracteres (a sequência entre parênteses) foram excluídos posteriormente. Observe-se que as exclusões devem ser lidas na direção inversa, ou seja, a leitura de "[rimrod_arap_sanicidem $]$ " de trás para frente informa a cadeia de caracteres excluída. Mesmo que "medicinas para dormir" e "tranquilizantes" sejam paráfrases, a primeira cadeira de caracteres excluída é parte da $\mathrm{AU}_{44}$; e a segunda, da $\mathrm{AU}_{45}$. A associação de múltiplas palavras excluídas àquelas encontradas no texto final para as quais elas de alguma forma contribuíram só pode ser aproximada, de modo que se deve esperar uma margem de erro para as palavras vizinhas. Seguindo Alves e Vale (2011), referimo-nos a essas revisões como microunidades, as quais serão discutidas na Seção 4.2 .

O tempo necessário para digitar a tradução é fornecido pelo atributo Dur. No exemplo anterior, mais de $11 \mathrm{~s}(11.407 \mathrm{~ms})$ foram necessários para todas as atividades de digitação na $\mathrm{AU}_{44}$, enquanto a digitação de "tranquilizantes", na $\mathrm{AU}_{44}$, precisou de $1.610 \mathrm{~ms}$.

A Tabela 8 exibe o tempo total de leitura (TrtS e TrtT) e o número de fixações (FixS e FixT) nos itens-fonte e nos itens-alvo. De acordo com essa informação, a SAU da palavra "of", na $\mathrm{AU}_{44}$, foi fixada duas vezes, com um tempo total de leitura igual a $167 \mathrm{~ms}$, enquanto a tradução "de" foi fixada 12 vezes, com um tempo total de $1.232 \mathrm{~ms}$. A cadeia de caracteresfonte na $\mathrm{AU}_{45}$ foi fixada 27 vezes, com um TrtS de $1.896 \mathrm{~ms}$, ao passo que a cadeia de caracteres-alvo recebeu oito fixações, com um TrtT de $615 \mathrm{~ms}$.

\subsection{Microunidades}

Alves e Vale (2011) denominam microunidades as atividades recorrentes de edição para a tradução de uma mesma palavra ou grupo de palavras. Para Alves e Vale (2011, p. 107), "define-se uma microunidade de tradução como o fluxo de produção contínua do 
texto-alvo [...] separado por pausas durante o processo tradutório". Por sua vez, uma macrounidade consiste em um conjunto de microunidades "que compreende todas as produções de texto provisórias que correspondem ao foco do tradutor em um mesmo segmento do texto-fonte" (ALVES; VALE 2011, p. 107).

O TPR-DB computa as unidades de "produção contínua do texto-alvo" como unidades de produção (cf. Seção 4.5) e lista, nas tabelas, os detalhes das duas primeiras microunidades que contribuíram para a produção de dada tradução. A coluna Munit da Tabela 8 indica quantas microunidades contribuíram para a produção de uma AU. Embora possa haver, a princípio, qualquer número de microunidades - um tradutor pode revisar dada parte de um texto com frequência -, indicam-se, conforme a seguir, as informações detalhadas apenas das duas primeiras microunidades.

As Tabelas 9 e 10 exibem as informações das microunidades da $\mathrm{AU}_{44}$ e da $\mathrm{AU}_{45}$.Uma microunidade conta com informações sobre o tempo inicial (Time) e sobre a duração (Dur) da atividade de sua digitação, um valor de pausa que precede essa atividade (Pause) e a quantidade de atividade de leitura concomitante no texto-fonte (ParalS) e no texto-alvo (ParalT). E o importante: uma microunidade é caracterizada pela atividade real de digitação, representada pela cadeia na coluna Edit.

Tabela 9 - Primeiras microunidades referentes a "tranquilizantes"

\begin{tabular}{c|c|c|c|c|c|c|c|c}
\hline AUid & Edit1 & Time1 & Dur1 & Pause1 & FixS1 & ParalS1 & FixT1 & ParalT1 \\
\hline 44 & de_medicinas_para_dormir & 225.703 & 11.110 & 187 & 10 & 716 & 2 & 116 \\
\hline 45 & tranquilizantes & 570.250 & 1.610 & 172 & 0 & 0 & 9 & 536 \\
\hline
\end{tabular}

Tabela 10 - Microunidades 1 e 2

\begin{tabular}{c|c|c|c|c|c|c|c|c}
\hline AUid & Edit2 & Time2 & Dur2 & Pause2 & FixT2 & ParalS2 & FixT2 & ParalT2 \\
\hline 44 & [rimrod_arap_sanicidem] & 569.781 & 297 & 22.937 & 0 & 0 & 4 & 214 \\
\hline 45 & - & 0 & 0 & 0 & 0 & 0 & 0 & 0 \\
\hline
\end{tabular}

As Tabelas 9 e 10 decompõem a atividade de produção da Tabela 8 em duas microunidades: no tempo $225.703 \mathrm{~ms}$ (Time), o tradutor produziu a primeira microunidade na $\mathrm{AU}_{44}$, digitando "de medicinas para dormir"; no tempo $569.781 \mathrm{~ms}$, em uma fase de revisão que ocorreu mais de quatro minutos depois, na microunidade 2 (Tabela 10) foi excluída a cadeia de caracteres "medicinas para dormir", havendo, no tempo 570.250, sua substituição por "tranquilizantes", que é parte da microunidade 1 da $\mathrm{AU}_{45}$ (Tabela 9). A duração dessas 
atividades é fornecida juntamente com as pausas que as seguem e com as atividades concomitantes do olhar. Com base nas informações da Tabela 3, sabemos que a fase de revisão (TimeR) começou no tempo $290.391 \mathrm{~ms}$ da correspondente sessão de tradução. Sendo assim, observamos que a microunidade 1 da $\mathrm{AU}_{44}$ ocorre durante a fase de redação (drafting), enquanto a microunidade 2 da $A_{44}$ e a microunidade 1 da $A_{45}$ constituem eventos de revisão (revision).

\subsection{Ineficiência de edição}

A ineficiência de edição (InEff) mede a razão entre o número de caracteres produzidos e o comprimento da tradução final, o que corresponde, aproximadamente, à soma do número de inserções com o número de exclusões dividida pela diferença entre esses dois números, conforme explicitado na Equação Eq1.

(Eq1) InEff = número de caracteres digitados / comprimento da tradução final

$$
\approx(\text { inserções }+ \text { exclusões }) /(\text { inserções-exclusões })+1 .
$$

Na maioria dos casos, o comprimento de uma palavra equivale ao número de inserções de caracteres menos o número de exclusões de caracteres mais " 1 ”. Adiciona-se " 1 ” porque o espaço em branco após a palavra é contato como parte dela. No entanto, há vezes em que não há espaço em branco após a palavra; nesses casos, o valor de InEff pode ser menor que "1". Dessa forma, para a $\mathrm{AU}_{44}$, na Tabela 8, o somatório de inserções e exclusões é igual a 45, valor esse que, dividido por "3" - número de caracteres na palavra final "of" (incluindo o espaço em branco) -, resulta em uma ineficiência de edição igual a 15; por sua vez, o número de teclas digitadas em cadeia para produzir a palavra "tranquilizantes" na $\mathrm{AU}_{45}$ corresponde ao comprimento da tradução final, de modo que o esforço de edição é igual 0,94. Observe-se que, para a pós-edição, o valor de InEff pode ser zero se a proposta da tradução automática for aceita sem qualquer modificação, mas seria igual a " 2 " se a palavra fosse excluída e outra de comprimento idêntico fosse digitada novamente.

\subsection{Atributo Cross}

$\mathrm{O}$ atributo Cross representa as informações do alinhamento entre as palavras do textofonte e aquelas do texto-alvo como uma distorção local entre as línguas em questão. Essa distorção tem uma direção dependente, partindo do texto-fonte em direção ao texto-alvo 
(CrossS), ou vice-versa (CrossT). Talvez os valores desse atributo possam ser mais bem compreendidos como uma referência ao método de geração de uma sentença - da esquerda para a direita - por meio de ligações de alinhamento em que se contam quantas palavras devem ser "saltadas" em uma língua para se produzir a próxima palavra na outra língua.

A Figura 3 exemplifica uma tradução inglês $\rightarrow$ espanhol. Ela mostra duas sentenças alinhadas, os valores de Cross, a enumeração dos itens (tokens) nas duas sentenças e as ligações entre o texto-fonte e o texto-alvo. Para produzir a primeira palavra na tradução em espanhol ("El"), duas palavras em inglês ("Killer" e "nurse") tiveram de ser "saltadas" no texto-fonte, resultando em um valor Cross igual a "2". Como a segunda palavra no texto-fonte ("nurse") produz duas palavras adjacentes no texto-alvo, nenhuma palavra no texto-fonte deve ser "saltada" para produzir "enfermero", resultando em um valor Cross igual a zero. Para produzir a terceira palavra em espanhol, "asesino", uma palavra do texto-fonte, localizada à esquerda de "nurse", deve ser processada, levando ao valor Cross de "-1". Já a próxima palavra em espanhol, "recibe", é a tradução de duas palavras à direita da atual posição do cursor no texto-fonte, levando a um valor Cross igual a "2". Dessa forma, os valores TT Cross indicam uma reordenação relativa das palavras do texto-fonte para se chegar à tradução do texto-alvo.

Figura 3 - Valores Cross das unidades do texto-fonte e texto-alvo

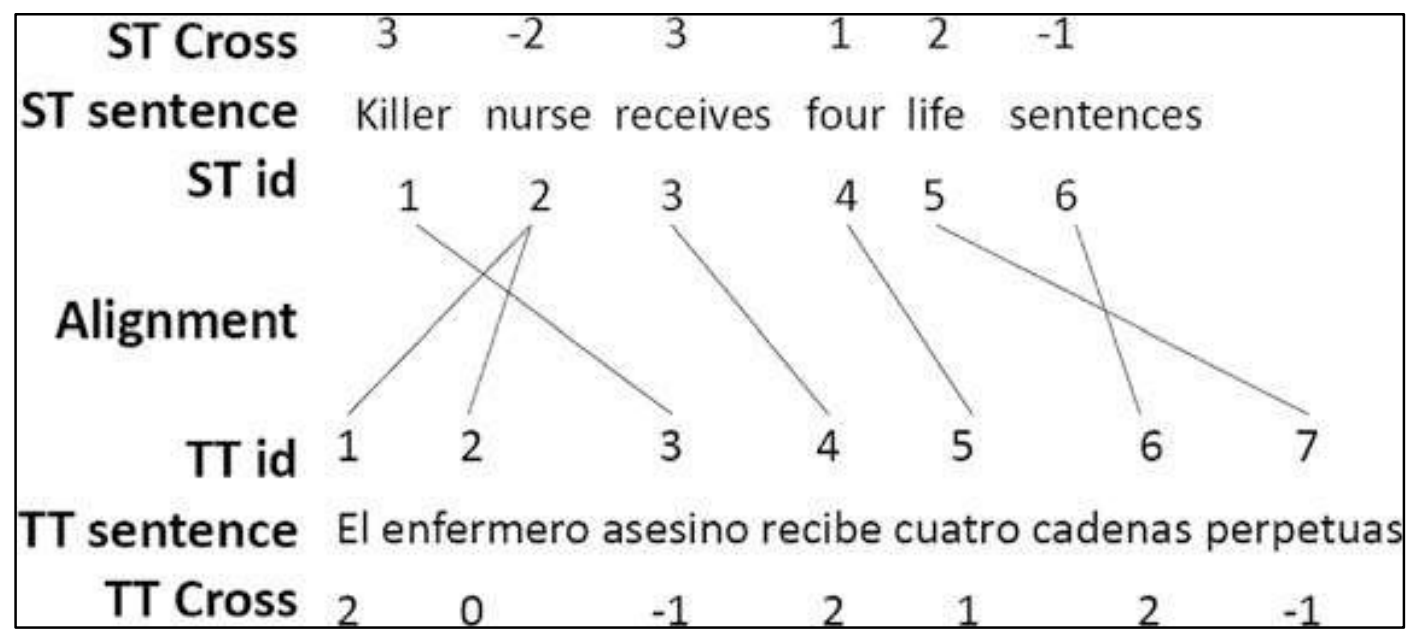


Também se computam valores de Cross para o texto-fonte. Os valores de ST Cross presumem que o texto-fonte é o texto de saída e o texto-alvo é o texto de entrada. Portanto, esses valores indicam a reordenação relativa das palavras do texto-alvo para se chegar ao texto-fonte.

Línguas com similaridade na ordem das palavras apresentam, em média, valores Cross baixos. Em uma tradução uniforme de "1" para "1", todos os valores Cross são iguais a "1". Quanto maior o número de reordenações sintáticas entre o texto-fonte e o texto-alvo, maior é a média dos valores do atributo Cross.

\subsection{Itens (tokens) do texto-fonte e do texto-alvo}

As tabelas de resumo dos itens do texto-fonte (ST) e dos itens do texto-alvo (TT) contêm basicamente os mesmos dados que aqueles presentes nas tabelas de AU. Em especial, as informações gerais, como o nome do estudo (Study) e o nome da sessão (Session), são idênticas. No entanto, no lugar dos atributos SAL e TAU (como vistos na Tabela 6), as tabelas ST e TT apresentam os atributos SToken e TToken, bem como uma etiquetagem de Lemma (lemas) e outra de PoS (classes gramaticais - do inglês, part of speech) dos itens da línguafonte e da língua-alvo, respectivamente.

A Tabela 11 apresenta três itens do texto-fonte. Os atributos Prob1e Prob2 são probabilidades, em $\log _{10}$, de monogramas e bigramas. No caso do inglês, utilizou-se o $\mathrm{BNC}^{12}$ como corpus de referência. No caso, existe uma probabilidade de $10^{-3,4339}=1 \mathrm{em} 2.715$ e de $10^{-6,1669}=1$ em 1.468 .588 para a ocorrência de, respectivamente, "four" e de "life sentence" no BNC.

Tabela 11 - Informação dos itens do texto-fonte (ST)

\begin{tabular}{c|c|c|c|c|c|c|c|c|c|c|c}
\hline STid & STseg & Study & Session & $\ldots$ & SToken & Lemma & Prob1 & Prob2 & PoS & TToken & TTid \\
\hline 5 & 1 & BML12 & P01_T1 & $\ldots$ & four & four & $-3,4339$ & -50 & CD & cuatro & 5 \\
\hline 6 & 1 & BML12 & P01_T1 & $\ldots$ & life & life & $-3,3508$ & -50 & NN & perpetuas & 7 \\
\hline 4 & 1 & BML12 & P01_T1 & $\ldots$ & sentences & sentence & $-4,64$ & $-6,1669$ & NNS & cadenas & 6 \\
\hline
\end{tabular}

Também existem mais informações detalhadas sobre o olhar nas tabelas ST e TT. Além disso, as tabelas ST contêm informações sobre a entropia na tradução das palavras.

12 BRITISH NATIONAL CORPUS. Disponível em: <http://www.natcorp.ox.ac.uk/>. Acesso em: 16 nov. 2017. 


\subsection{Informação do olhar (gaze)}

Diversas novas medidas de tempo de leitura foram adicionadas ao TPR-DB 2.0. Seguimos sugestões propostas pelo Kertz $\mathrm{Lab}^{13}$, as quais foram implementadas da seguinte maneira:

- FFTime, ou tempo da primeira fixação (do inglês, first fixation time): é o tempo (em ms) despendido até a primeira fixação em dado item (token);

- FFDur, ou duração da primeira fixação (do inglês, first fixation duration): é a duração da primeira fixação no item;

- FPDurS, ou tempo de leitura do item-fonte quando da primeira passagem por ele (do inglês, first pass source token reading duration): é a soma das durações de todas as fixações no item-fonte desde a primeira fixação até o momento em que os participantes olham para um item diferente;

- FPDurT ou tempo de leitura do item-alvo quando da primeira passagem por ele (do inglês, first pass target token reading duration): a soma das durações de todas as fixações no item-alvo desde a primeira fixação até o momento em que os participantes olham para um item diferente;

- RPDur, ou duração do percurso de regressão (do inglês, regression path duration): é a quantidade de tempo necessária desde o FFTime até que os olhos se movam para a direita no texto (em outras palavras, inclui todas as regressões para a esquerda);

- Regr, ou regressão (do inglês, regression), é um valor booleano que indica se uma regressão sucedeu a primeira passagem de leitura;

- FixS, ou número total de fixações no item-fonte (do inglês, fixations on the source token);

- FixT, ou número total de fixações no item-alvo (do inglês, fixations on the target token);

- TrtS, ou tempo total de leitura no item-fonte (do inglês, total reading time on source token): é a soma de todas as fixações no item-fonte em toda a sessão;

- TrtT, ou tempo total de leitura no item-alvo (do inglês, total reading time on

13 KERTZ LAB. Disponível em: $<$ https://wiki.brown.edu/confluence/display/kertzlab/EyeTracking+While+Reading>. Acesso em: 17 nov. 2017. 
target token): é a soma de todas as fixações no item-alvo em toda a sessão.

A Tabela 12 exibe exemplos dessas medições do olhar. De acordo com as definições, FFDur é sempre menor que RPDur ou Trt.

Tabela 12 - Informações do olhar nas unidades do texto-fonte e texto-alvo (parte 1)

\begin{tabular}{c|c|c|c|c|c|c|c|c|c|c|c|c|c}
\hline STid & Study & Session & $\ldots$ & FFTime & FFDur & RPDur & Regr & FixS & FPDurS & TrtS & FixT & FPDurT & TrtT \\
\hline 4 & BML12 & P01_T1 & $\ldots$ & 280 & 50 & 1317 & 1 & 9 & 50 & 1567 & 3 & 133 & 183 \\
\hline 5 & BML12 & P01_T1 & $\ldots$ & 1843 & 650 & 650 & 0 & 8 & 650 & 1600 & 24 & 149 & 1945 \\
\hline 6 & BML12 & P01_T1 & $\ldots$ & 2515 & 283 & 866 & 1 & 12 & 666 & 1498 & 0 & 0 & 0 \\
\hline
\end{tabular}

\subsection{Entropia e perplexidade de tradução}

A perplexidade de tradução indica quantas escolhas de tradução um tradutor tem para determinado ponto do texto-fonte, ou seja, quantas palavras igualmente prováveis podem ser produzidas para certa palavra-fonte em dado contexto. Nós assumimos que a escolha das traduções segue certa distribuição de probabilidades de tradução $\boldsymbol{p}$ e estimamos essas probabilidades a partir de um corpus de traduções alinhadas. As probabilidades de tradução $p\left(s \rightarrow t_{i}\right)$ para dada palavra $s$ do texto-fonte e suas possíveis correspondências na tradução $\boldsymbol{t}_{\boldsymbol{i} . . .} \boldsymbol{n}$ são computadas como a razão entre o número de alinhamentos $\boldsymbol{s} \rightarrow \boldsymbol{t}_{\boldsymbol{i}}$ contados nos textos-alvo e o número total de itens observados no texto-alvo, conforme mostra a Equação Eq2.

(Eq2) $p\left(s \rightarrow t_{\mathrm{i}}\right)=\frac{\text { número de }\left(s \rightarrow t_{\mathrm{i}}\right)}{\text { número de traduções }}$

A informação de uma distribuição com probabilidades iguais, $\boldsymbol{p}$, é definida por $\boldsymbol{I}(\boldsymbol{p})=-\log _{2}(\boldsymbol{p})$. Enquanto a probabilidade expressa a expectativa para um evento, essa informação indica a quantidade mínima de bits com a qual essa expectativa pode ser codificada. A entropia $\boldsymbol{H}$ indica a expectativa para essa informação, também entre distribuições de probabilidades desiguais, conforme visto na Equação Eq3.

(Eq3) $H(s)=\sum_{i=1}^{n} p\left(s \rightarrow t_{\mathrm{i}}\right)^{*}-\log _{2}\left(p\left(s \rightarrow t_{i}\right)\right)$

A entropia de tradução, $\boldsymbol{H}(\mathbf{s})$, é a soma de todas as probabilidades (i.e., expectativas) 
observadas para a tradução de dada palavra-fonte, $\boldsymbol{s}$, nas palavras-alvo, $\boldsymbol{t}_{\boldsymbol{i}} \ldots \boldsymbol{n}$, multiplicada pelo seu conteúdo informacional. Isso representa a quantidade média de informações contidas em uma escolha de tradução. Sendo assim, se dada palavra-fonte $s$ tiver somente uma possibilidade de tradução $t$ em determinado contexto, sua tradução é $p(s \rightarrow t)=1$, sua informação é $I(p(s \rightarrow t))=0$ bit e, portanto, sua entropia $H(s)=0$ é mínima. Quanto mais traduções diferentes igualmente prováveis uma palavra-fonte tiver, maior será sua entropia $\boldsymbol{H}(\mathbf{s})$ de tradução.

A perplexidade $(\boldsymbol{P P})$ está relacionada com a entropia $\boldsymbol{H}$ seguindo uma função exponencial, conforme revelado na Equação Eq4.

(Eq4) $P P(s)=2^{H(s)}$

Quanto maior for a perplexidade, maior será a probabilidade de existirem escolhas semelhantemente possíveis e, portanto, mais difícil será a tomada de decisão.

As tabelas ST fornecem algumas dessas informações. CountT representa o número de alinhamentos $\left(\mathrm{s} \rightarrow \mathrm{t}_{\mathrm{i}}\right)$ observados em SToken $\rightarrow$ TToken $_{\mathrm{i}}$ alignments count. AltT aponta o número de diferentes TTokens ${ }_{\mathrm{i}}$. ProbT refere-se à probabilidade de ocorrência do item. HTra é a entropia de tradução do SToken.

Por exemplo, considere-se $\mathrm{STid}_{4}$ na Tabela 13. A tradução "four $\rightarrow$ cuatro" ocorreu 25 vezes no corpus, com uma probabilidade de 0,8 . Com isso, podemos reconstruir o número total de traduções no corpus como sendo $31 \approx 25 / 0,8$, sendo que as seis traduções restantes $(31-25)$ foram distribuídas como três palavras diferentes.

Tabela 13 - Informações do olhar nas unidades do texto-fonte e texto-alvo (parte 2)

\begin{tabular}{c|c|c|c|c|c|c|c|c|c|c|c|c}
\hline STid & Study & Session & $:::$ & SToken & TToken & SAUnbr & TAUnbr & AltT & CountT & ProbT & HTra & HSeg \\
\hline 4 & BML12 & P01_T1 & $:::$ & four & cuatro & 1 & 1 & 4 & 25 & 0,8065 & 0,9511 & 0,7088 \\
\hline 5 & BML12 & P01_T1 & $:::$ & life & perpetuas & 1 & 1 & 8 & 17 & 0,5484 & 1,9385 & 0,6595 \\
\hline 6 & BML12 & P01_T1 & $:::$ & sentences & cadenas & 1 & 1 & 8 & 18 & 0,5806 & 1,899 & 0,4587 \\
\hline
\end{tabular}

O atributo HSeg indica a entropia da segmentação do alinhamento das palavras. Por exemplo, uma expressão como "life sentences" poderia ser alinhada como uma única unidade multipalavra ou como duas unidades distintas. O número de palavras da língua-fonte e da 
língua-alvo da unidade de alinhamento $(\mathrm{AU})$ da qual "life" faz parte está refletido nos valores SAUnbr e TAUnbr, respectivamente. O atributo HSeg leva em consideração esse contexto de segmentação do alinhamento, sendo calculado de forma parecida com o atributo HTra, mas com a diferença de que depende da contagem de TAUnbr idênticos, em vez da contagem de TToken.

O atributo Literal, na Tabela 5, é, pois, basicamente a literalidade média na tradução das palavras. É calculada por Literal $=\frac{1}{n} * \sum_{j}^{n} \operatorname{abs}\left(\operatorname{cross}^{*} \boldsymbol{H} \operatorname{tra}\right)$, em que $\boldsymbol{n}$ é o comprimento da sentença do texto-fonte.

\section{Unidades de processamento}

Esta seção começa com a descrição das unidades básicas de processamento, dados de acionamentos únicos de teclas (KD - em inglês, keystroke data) e de fixações (FD - em inglês, fixation data). As Seções 5.3 e 5.4 introduzem, respectivamente, as unidades de produção (PU - em inglês, production units) e as unidades de fixação (FU - em inglês, fixation units). A Seção 5.5 apresenta a noção de unidades de atividade (CU - em inglês, activity units), que fragmenta exaustivamente o processo tradutório em oito tipos de segmentos.

\subsection{Dados dos acionamentos de teclas}

Dentro do TPR-DB, cada acionamento de tecla feito por um tradutor humano é caracterizado por sete elementos, quais sejam:

1. Time: o tempo (ms) despendido até o acionamento de tecla;

2. Type: a distinção entre os tipos acionamentos (de inserção e de exclusão);

3. Cursor: a posição do texto-alvo em que se dá o acionamento de tecla;

4. Char: o caractere (UTF8) que é alvo do acionamento (para inserção ou exclusão);

5. TTseg: o segmento-alvo (sentença-alvo) que está sendo produzido(a);

6. STid: a identificação da palavra do texto-fonte da qual a palavra-alvo produzida é uma tradução;

7. TTid: a identificação da palavra do texto-alvo que está sendo processada pelo acionamento de tecla.

O exemplo da Tabela 14 mostra os dados de acionamentos de teclas para a produção 
de duas palavras em espanhol, "El enfer[e]mero", como tradução da palavra-fonte STid $_{2}$. Essas são as duas primeiras palavras do primeiro segmento da tradução. A tabela registra somente os acionamentos de teclas para modificação do texto, ou seja, para inserções e exclusões - as informações de navegação, como cliques do mouse, são ignoradas. As inserções e exclusões podem ser produzidas manualmente (Mins e Mdel) ou automaticamente (Ains e Adel). A Tabela 14 contém, na linha 9, um exemplo de exclusão manual.

Tabela 14 - Informações de acionamentos de teclas na sessão P01_T1 do estudo BML12

\begin{tabular}{|c|c|c|c|c|c|c|c|}
\hline KDid & Time & Type & Cursor & Char & TTseg & STid & TTid \\
\hline 0 & 92.016 & Mins & 0 & “E” & 1 & 2 & 1 \\
\hline 1 & 92.172 & Mins & 1 & "1" & 1 & 2 & 1 \\
\hline 2 & 92.313 & Mins & 2 & “" & 1 & 2 & 1 \\
\hline 3 & 92.375 & Mins & 3 & "e" & 1 & 2 & 2 \\
\hline 4 & 92.563 & Mins & 4 & "n” & 1 & 2 & 2 \\
\hline 5 & 92.828 & Mins & 5 & "f" & 1 & 2 & 2 \\
\hline 6 & 92.938 & Mins & 6 & "e" & 1 & 2 & 2 \\
\hline 7 & 93.047 & Mins & 7 & "r" & 1 & 2 & 2 \\
\hline 8 & 93.266 & Mins & 8 & "e" & 1 & 2 & 2 \\
\hline 9 & 93.610 & Mdel & 8 & "e" & 1 & 2 & 2 \\
\hline 10 & 93.797 & Mins & 8 & “m” & 1 & 2 & 2 \\
\hline 11 & 93.875 & Mins & 9 & "e" & 1 & 2 & 2 \\
\hline 12 & 93.938 & Mins & 10 & "r" & 1 & 2 & 2 \\
\hline 13 & 94.078 & Mins & 11 & “o” & 1 & 2 & 2 \\
\hline 14 & 94.203 & Mins & 12 & “ & 1 & 2 & 2 \\
\hline
\end{tabular}

\subsection{Dados de fixação}

Durante a fixação Dentro do TPR-DB, o centro de uma fixação é mapeado sobre o caractere mais próximo na tela e conectado aos dez atributos seguintes:

1. Time: momento em que a fixação teve início;

2. Dur: duração da fixação em ms;

3. Win: janela-fonte (1) ou janela-alvo (2) na qual a fixação é observada;

4. Cursor: mapeamento do centro da fixação no caractere mais próximo na janela;

5. STid: identificação do item (token) do texto-fonte que está sendo visualizado;

6. TTid: identificação do item do texto-alvo que está sendo visualizado;

7. Seg: identificação do segmento da palavra do texto-fonte (STid) que está sendo 
visualizada;

8. ParalK: quantidade de atividades de digitação concomitante, ou seja, unidades de produção (PU, cf. Seção 5.3);

9. Edit: caractere(s) digitado(s) durante a fixação;

10. EDid: identificação do segmento-alvo produzido pela digitação dos caracteres.

A Tabela 15 mostra uma sequência de 13 fixações, FDid 507-519, que fazem parte da sessão P01_T1, introduzida anteriormente. Todas as fixações ocorrem na janela 1, no primeiro segmento e nos itens de STid 4, 6, 3 e 5, traduzidos, respectivamente, nos itens de TTid 5, 6, 4 e 7. Algumas fixações apresentam atividades de digitação concomitante: como a quantidade de atividade paralela de teclado (ParalK) equivale ao tempo de duração das fixações (Dur), as sete primeiras fixações (FDid 507-513) se sobrepõem a $100 \%$ da produção do texto. Não ocorreu atividade no teclado durante as fixações FDid 515-517, mas uma sobreposição parcial de 16\% (124 ms/750 ms) foi encontrada entre a atividade de digitação e a fixação FDid 518. Durante as fixações 507-510, por exemplo, foi digitada (Edit) a sequência "eno", que é parte da produção de "asesino". A coluna EDid indica o número de identificação STid da tradução produzida, ou seja, "asesino" é uma tradução de STid 3.

Tabela 15 - Informações de fixação (.fd file)

\begin{tabular}{c|c|c|c|c|c|c|c|c|c|c}
\hline FDid & Time & Dur & Win & Cursor & Seg & STid & TTid & ParalK & Edit & EDid \\
\hline 507 & 94.530 & 150 & 1 & 25 & 150 & 4 & 5 & 150 & $\mathrm{e}$ & $3 \mathrm{C}$ \\
\hline 508 & 94.749 & 67 & 1 & 24 & 1 & 4 & 5 & 67 & - & - \\
\hline 509 & 95.077 & 67 & 1 & 25 & 1 & 4 & 5 & 67 & $\mathrm{n}$ & $3 \mathrm{C}$ \\
\hline 510 & 95.218 & 67 & 1 & 26 & 1 & 4 & 5 & 67 & $\mathrm{o}$ & $3 \mathrm{C}$ \\
\hline 511 & 98.952 & 50 & 1 & 36 & 1 & 6 & 6 & 50 & $\mathrm{i}$ & $4 \mathrm{C}$ \\
\hline 512 & 99.015 & 167 & 1 & 37 & 1 & 6 & 6 & 167 & $\mathrm{~b}$ & $4 \mathrm{C}$ \\
\hline 513 & 99.202 & 50 & 1 & 36 & 1 & 6 & 6 & 50 & - & - \\
\hline 514 & 99.265 & 83 & 1 & 25 & 1 & 4 & 5 & 1 & $\mathrm{e}$ & $4 \mathrm{C}$ \\
\hline 515 & 99.499 & 100 & 1 & 16 & 1 & 3 & 4 & 0 & - & - \\
\hline 516 & 99.624 & 83 & 1 & 16 & 1 & 3 & 4 & 0 & - & - \\
\hline 517 & 99.718 & 50 & 1 & 17 & 1 & 3 & 4 & 0 & - & - \\
\hline 518 & 99.780 & 750 & 1 & 24 & 1 & 4 & 5 & 124 & - & $4 \mathrm{C}$ \\
\hline 519 & 100.546 & 250 & 1 & 30 & 1 & 5 & 7 & 250 & - & - \\
\hline
\end{tabular}

$\mathrm{Na}$ Seção 5.3, mostramos que a sequência de teclas é parte de uma unidade de produção, $\mathrm{PU}$ o, enquanto as fixações são parte de $\mathrm{FU}_{14}$. A Seção 6 dispõe os dados em um 
contexto mais amplo.

\subsection{Unidades de produção}

As unidades de produção (PU) são sequências de atividades contínuas de digitação. De acordo com a definição em Carl e Kay (2011), a delimitação de uma PU é definida como uma pausa de $1000 \mathrm{~ms}$ ou mais sem qualquer atividade no teclado. Presume-se que uma pausa superior a essa indique uma interrupção no fluxo de digitação, com um possível desvio da atenção em direção a um segmento diferente do texto. Como um segmento temporal/textual contínuo, as PU possuem um tempo inicial (Time) e uma duração (Dur) e podem englobar um ou mais acionamentos de teclas para inserção ou exclusão (Edit) que contribua para a produção de um ou mais itens do texto-alvo (TTid). A Tabela 16 disponibiliza três unidades de produção, uma das quais, $\mathrm{PU}_{0}$, tem sua sequência de edição apresentada no exemplo E1.

Tabela 16 - Três unidades de produção da sessão P01_T1 do estudo BML12

\begin{tabular}{c|c|c|c|c|c|c|c|c}
\hline PUid & Estudo & Sessão & Time & Dur & Pausa & Ins & Del & Edit \\
\hline 0 & BML12 & P01_T1 & 92.016 & 7250 & 92.016 & 34 & 7 & $\begin{array}{c}\text { El_enfere[e]mero_ } \\
\text { asesiono_re[er_ono]no_recibe }\end{array}$ \\
\hline 1 & BML12 & P01_T1 & 100.406 & 1313 & 1140 & 8 & 0 & _cuatro_ \\
\hline 2 & BML12 & P01_T1 & 103.594 & 4187 & 1875 & 23 & 3 & $\begin{array}{c}\text { sentencias_de_vida. } \\
\text { _[_.]_ }\end{array}$ \\
\hline
\end{tabular}

(E1) El_enfere [e] mero_asesiono_re [er_ono] no_recibe

E1 iniciou no tempo $92.016 \mathrm{~ms}$ e foi digitado em $7250 \mathrm{~ms}$, sem interrupções superiores a $1000 \mathrm{~ms}$ entre os acionamentos das teclas. Esse exemplo foi precedido por uma pausa de $92.016 \mathrm{~ms}$.

A próxima unidade, $\mathrm{PU}_{1}$, começou com uma pausa de $1140 \mathrm{~ms}$. Após essa pausa, a sequência de digitação começa no tempo 100.406 ms e dura $1.313 \mathrm{~ms}$.

A Tabela 16 indica o número de inserções e exclusões das PUs. A unidade PU。 contém 34 inserções (Ins) e sete exclusões (Del). As exclusões estão dentro de colchetes na coluna Edit, e devem ser lidas de trás para frente. Dessa forma, a subsequência "[er_ono]" na verdade reflete a exclusão "ono_re", conforme visto no exemplo E2. 
asesiono_re $\rightarrow$ asesino_recibe

A Tabela 17 - que na verdade é uma continuação da Tabela 16 - contém informações adicionais do produto e do processo das três PUs. STseg e TTseg indicam que as três PU fazem parte da tradução do primeiro segmento. STid e TTid exibem as palavras-alvo e as palavras-fonte abrangidas pela tradução. Observe-se que TTid se refere às numerações das palavras na tradução final; sendo assim, a numeração de uma palavra em uma versão intermediária do texto pode não coincidir com aquela no texto final se houver inserção ou exclusão de palavras.

Tabela 17 - Três unidades de produção da sessão P01_T1 do estudo BML12

\begin{tabular}{c|c|c|c|c|c|c|c|c|c|c|c|c|c}
\hline STsegTTseg & STid & TTid & FixS ParalSFixT & ParalT & Scatter & CrossSCrossT & PosS & PosT \\
\hline 1 & 1 & $\begin{array}{c}1+2 \\
+3\end{array}$ & $\begin{array}{c}1+2+ \\
3+4\end{array}$ & 10 & 735 & 0 & 0 & 0 & 2,67 & 1,25 & $\begin{array}{c}\text { NNP + VBP } \\
+ \text { NNS }\end{array}$ & $\begin{array}{c}\text { ART + NC + } \\
\text { NC + VLfin }\end{array}$ \\
\hline 1 & 1 & $3+4$ & $4+5$ & 4 & 504 & 0 & 0 & 0 & 2 & 1,5 & NNS + CD & VLfin + CARD \\
\hline 1 & 1 & $4+5$ & $5+7$ & 4 & 216 & 0 & 0 & 1 & 1,5 & 1 & CD + NN & CARD + ADJ \\
\hline
\end{tabular}

Conforme podemos constatar na sucessão da STid, a tradução se desenvolve sucessivamente na ordem das palavras do texto-fonte. A PU “ “cuatro_" diz respeito a duas palavra-fonte e a duas palavra-alvo $\left(\mathrm{STid}_{3+4}\right.$ e $\left.\operatorname{TTid}_{4+5}\right)$, dado que o espaço em branco representado pelo subtraço "_" - já conta como parte da próxima palavra. A PU 2 também compreende duas palavras, $\mathrm{TTid}_{5}+_{7}$, mesmo que a PU seja constituída por três palavras "sentencias de vida", haja vista que o grupo nominal foi reescrito posteriormente como “cadenas perpetuas", formando $\mathrm{TTid}_{6}$ e $\mathrm{TTid}_{7}$. Observe-se que essa descontinuidade também é o motivo pelo qual o valor de Scatter é igual a "1": há uma sequência de dois acionamentos sucessivos de teclas nessa PU que produz traduções separadas por mais de uma palavra.

Os atributos FixS e FixT representam os números de fixações contadas, respectivamente, no lado-fonte e no lado-alvo das PU. Observe-se que, devido a uma má qualidade do rastreamento ocular, nenhuma fixação foi registrada nas cadeias de caracteres do texto-alvo.

Os atributos ParalS e ParalT indicam as quantidades de tempo que o tradutor estava olhando, respectivamente, para a janela-fonte e para a janela-alvo enquanto produzia a tradução. Em outras palavras, durante os $7.250 \mathrm{~ms}$ despendidos para produzir a $\mathrm{PU}_{0}$, o 
tradutor olhou por quase $1 \mathrm{~s}(900 \mathrm{~ms})$ para a janela do texto-fonte.

Os atributos CrossS e CrossT representam a média de distorção local entre o ladofonte e o lado-alvo das PU. O cálculo dos atributos Cross é discutido em detalhe na Seção 4.4. Os atributos PosS e PosT indicam as etiquetas de classes gramaticais das palavras-fonte e das palavras-alvo envolvidas nas PU.

\subsection{Unidades de fixação}

As unidades de fixação (FUs) descrevem sequências de comportamento de leitura contínua. Com base em evidências experimentais (CARL; KAY, 2011), definimos a delimitação entre duas FU sucessivas como uma pausa do olhar com duração superior a $400 \mathrm{~ms}$. Por exemplo, quando o olhar é desviado da tela por mais do que $400 \mathrm{~ms}$, interrompendo assim a atividade de leitura contínua, delimitamos uma unidade de fixação. Uma FU tem um tempo inicial e uma duração, sendo seguida por uma pausa (com duração superior $400 \mathrm{~ms}$ ) antes do início da FU subsequente.

$\mathrm{O}$ atributo Path descreve a sequência de palavras visualizadas na janela-fonte (1) ou na janela-alvo (2). O percurso do olhar consiste em uma ou mais fixações indicadas por uma sequência de variáveis "Win:WordID” com as fixações sucessivas sendo separadas por "+". A $\mathrm{FU}_{14}$, na Tabela 18, possui um caminho de quatro fixações $(1: 4+1: 4+1: 4+1: 4+)$ na palavra-fonte "four" (1:4). Por sua vez, a FU 15 , apresentada na Figura 4, representa um padrão de leitura no que diz respeito às palavras em negrito no título "Killer nurse receives four life sentences". Essa figura mostra como o olhar vai e volta entre as quatro palavras, o que levou $1844 \mathrm{~ms}$.

Tabela 18 - Quatro unidades de fixação

\begin{tabular}{c|c|c|c|c|c}
\hline FUid & Time & Dur & Pause & ParalK & Path \\
\hline 14 & 94.530 & 755 & 5293 & 755 & $1: 4+1: 4+1: 4+1: 4+$ \\
\hline 15 & 98.952 & 1844 & 3667 & 704 & $1: 6+1: 6+1: 6+1: 4+1: 3+1: 3+1: 3+1: 4+1: 5+$ \\
\hline 16 & 101.577 & 1272 & 781 & 142 & $1: 5+1: 5+1: 6+1: 6+1: 6+1: 5+1: 5+$ \\
\hline
\end{tabular}

Figura 4 - Captura de tela da repetição da situação $F U_{13}$ 


\section{trit Translog-II Replay [P01_T1.0m]] \\ Plot $0101.253 / 0757.281=13 \%$, " N W M Speed: $20 \%$ Go: $0098.720 \mathrm{~ms}$ Killer nurse receiles folages sentences \\ Hospital nurse Colin Notis was imprisoned for life toda! killed the four women in 2002 by giving them large am}

O atributo ParalK em uma tabela FU indica a quantidade de atividades paralelas de olhar e acionamento de teclas. Durante a $\mathrm{FU}_{14}$, o tradutor escreveu enquanto estava lendo. Já na $\mathrm{FU}_{16}$, houve uma sobreposição de $11 \%$ entre a atividade de digitação e a atividade de leitura.

Observe-se que a soma das durações de todas as FU pode ser maior que a soma das durações de todas as fixações. A razão é que as FU incluem intervalos interfixações com duração inferior a $400 \mathrm{~ms}$ que podem não integrar uma fixação.

\subsection{Unidades de atividade}

As unidades de atividade (CU) segmentam exaustivamente a sessão gravada em sequências de atividades que são levemente diferentes daquelas constantes nas tabelas PU e FU. Diferentemente dessas duas últimas, as CU dividem uma sessão em segmentos digitados. Para as tarefas de tradução, distinguem-se três tipos (em inglês, Types) de atividades do tradutor, a saber:

- tipo 1: leitura do texto-fonte;

- tipo 2: leitura do texto-alvo;

- tipo 4: digitação da tradução.

Como a leitura do texto-fonte ou do texto-alvo podem ocorrer em conjunto com a digitação (cf. Seção 4.3), também temos as seguintes atividades concomitantes:

- tipo 5: digitação da tradução concomitante com leitura do texto-fonte;

- tipo 6: digitação da tradução concomitante com leitura do texto-alvo;

- tipo 7: digitação da tradução concomitante com leitura do texto-fonte e do textoalvo.

Define-se uma atividade de digitação contínua como um uso ininterrupto do teclado 
(similar ao registrado nas PU), havendo apenas pausas iguais ou inferiores a $1 \mathrm{~s}$ entre dois acionamentos consecutivos de teclas. Se não forem registradas nem atividades do olhar nem atividades do teclado por mais do que $1 \mathrm{~s}$, tem-se um segmento ocioso:

- tipo 8: nenhuma atividade registrada.

Uma CU é descrita por seu tempo inicial (Time), duração (Dur) e segmento (Seg) no qual se encontra. A Figura 5 mostra uma sequência de quatro unidades de atividade envolvidas na tradução apresentada no Exemplo E3.

\section{E3 $\quad$ sleeping medicine $\rightarrow$ medicinas para dormir}

A Figura 5 mostra as delimitações das CUs e seus respectivos rótulos. A primeira CU, com a marcação temporal $231.500-232.500 \mathrm{~ms}$ é uma atividade de leitura do texto-fonte de $894 \mathrm{~ms}$, seguida por uma unidade "ociosa" (tipo 8) de $1107 \mathrm{~ms}$, na qual nenhuma atividade foi registrada. Em seguida, tem-se uma CU de digitação (Tipo 7), com início na marcação temporal de $233.500 \mathrm{~ms}$, com duração de $4640 \mathrm{~ms}$, na qual podem ser observadas leituras concomitantes do texto-fonte e do texto-alvo. Durante esse intervalo de tempo, foi produzida a tradução "medicinas para dormir A". Essa unidade foi seguida por uma atividade de leitura do texto-alvo (tipo 2, com duração de $484 \mathrm{~ms}$ ), na qual houve monitoramento da palavra recém-digitada (“dormir"). A figura representa um gráfico de progressão da tradução (do inglês, translation progression graph, ou TPG), que será discutido na Seção 6.

Figura 5 - Segmentação em unidades de atividades sucessivas

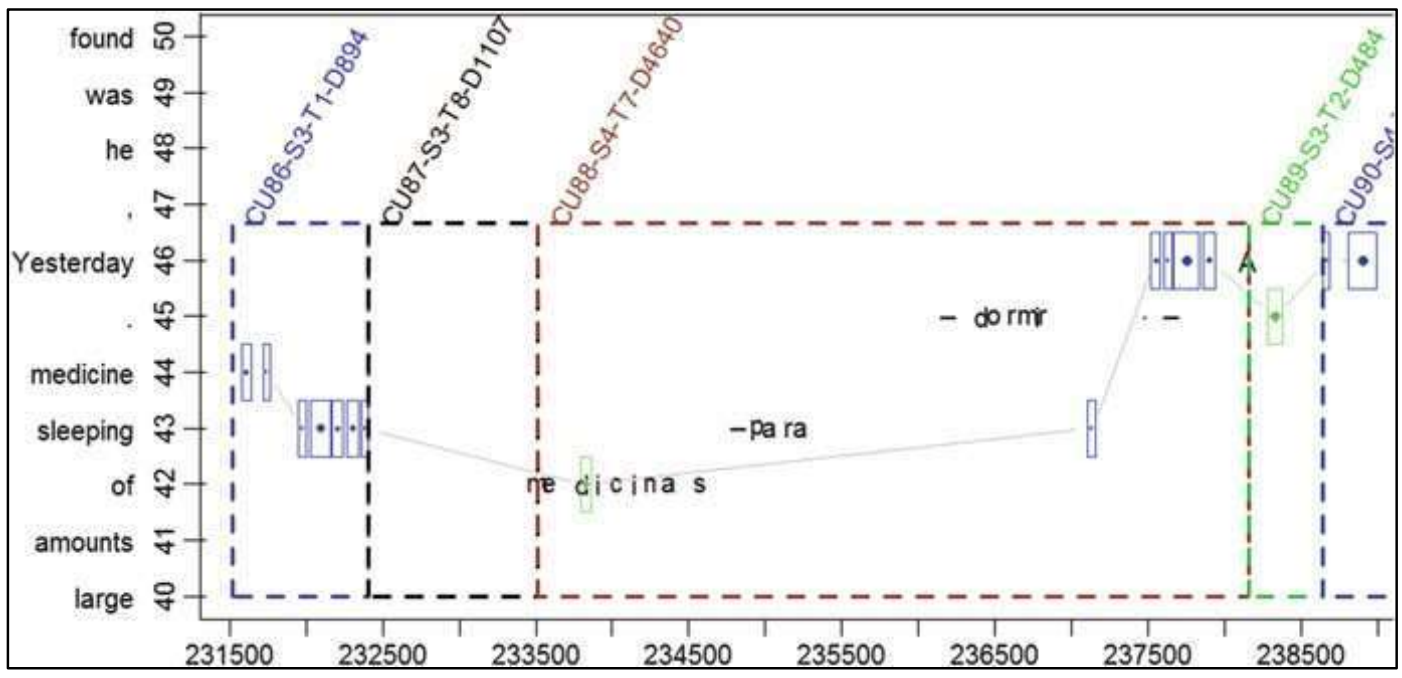




\section{Visualização dos dados do produto e do processo em um gráfico de progressão}

As informações de várias tabelas podem ser analisadas, avaliadas e visualizadas de diferentes formas. O gráfico de progressão da tradução (TPG) é um método de visualização que faz parte do TPR-DB. Os TPG permitem visualizar a forma como as traduções emergem ao longo do tempo, dispondo, de uma só vez, as informações parciais de diversas tabelas. A Figura 6 exibe um TPG para uma sessão de pós-edição no CASMACAT.

Figura 6 - Gráfico de progressão da tradução dispondo informações do olhar e dos acionamentos de teclas

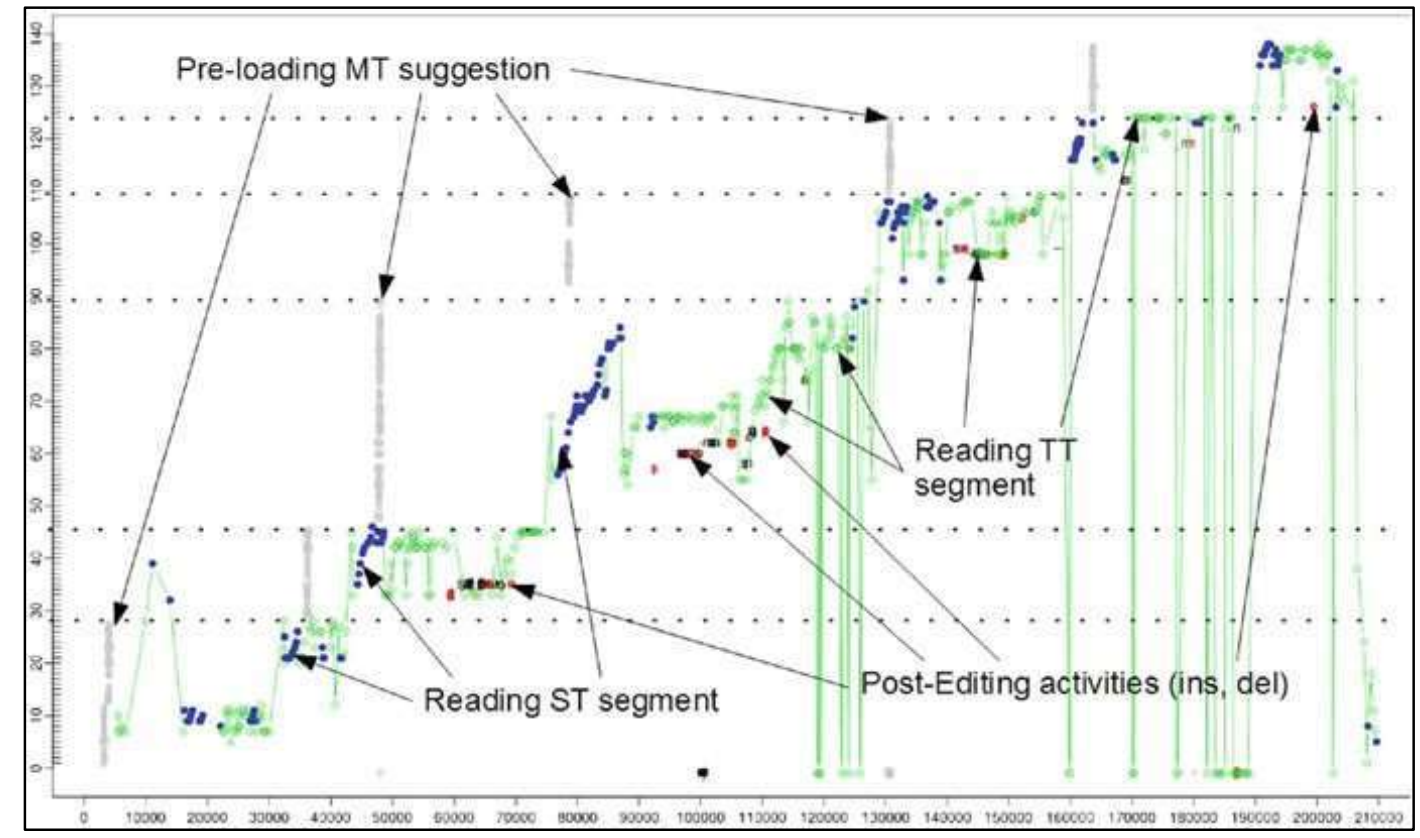

O gráfico esboça as atividades de pós-edição de seis segmentos consecutivos. O eixo vertical enumera as palavras do texto-fonte $(0 \ldots$. 140) com traços horizontais separando os segmentos, enquanto o eixo horizontal exibe o momento em que a tradução foi produzida para o respectivo texto-fonte. Os símbolos do gráfico são os seguintes:

- losangos azuis: representam as fixações no texto-fonte; 
- losangos verdes: representam as fixações no texto-alvo;

- caracteres pretos: representam as inserções;

- caracteres cinzas: representam as inserções automáticas;

- caracteres vermelhos: representam as exclusões.

O gráfico mostra quando os segmentos são traduzidos da língua-fonte para a línguaalvo, quando e onde o tradutor leu os segmentos-fonte e os segmentos-alvo e quando o texto foi modificado. Sendo assim, os gráficos de progressão são formas bastante úteis para avaliar os dados do TPR-DB quantitativamente.

Outro TPG é exibido na Figura 7. Esse gráfico relaciona o produto da tradução, considerando o texto-fonte (eixo vertical esquerdo) e o texto-alvo (eixo vertical direito), com os dados do processo tradutório em uma linha do tempo disposta no eixo horizontal, permitindo visualizar como a tradução emerge no tempo. As inserções são representadas por letras pretas, as exclusões estão em vermelho, e as fixações são pontos azuis dentro de retângulos que são mais largos ou mais estreitos conforme a duração.

Figura 7 - O gráfico de progressão apresenta as informações do produto e do processo das Tabelas 14 a 18

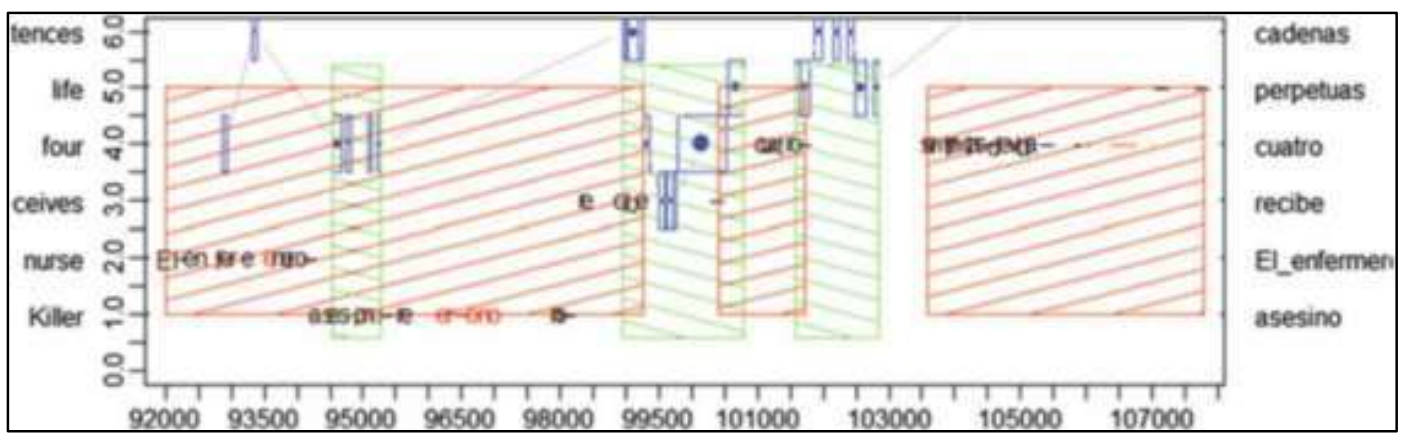

O TPG da Figura 7 esboça os dados dos acionamentos de teclas da Tabela 14, os dados de fixação da Tabela 15, bem como as três FU da Tabela 18 e as três PU das Tabelas 16 e 17. Os retângulos vermelhos preenchidos com traços indicam PU, enquanto os retângulos verdes preenchidos com traços indicam FU. A primeira parte (tempo aproximado de $92.000 \mathrm{~ms}$ até $94.000 \mathrm{~ms}$ ) reproduz a produção das palavras 1 e 2 ("El enfermero") como delineado na Tabela 14.

Conforme discutido nas Seções 5.3 e 5.4, as atividades de leitura e escrita podem ocorrer em paralelo. Por exemplo, a $\mathrm{FU}_{14}$, por volta do tempo $95.000 \mathrm{~ms}$, aconteceu enquanto o tradutor produzia a palavra "asesino", tradução de "Killer", ao passo em que a $\mathrm{FU}_{15}$ e a $\mathrm{FU}_{16}$, nas marcas temporais de, respectivamente, $99.000 \mathrm{~ms}$ e $101.500 \mathrm{~ms}$, se sobrepuseram 
apenas parcialmente à $\mathrm{PU}_{0}$ e à $\mathrm{PU}_{1}$ adjacentes. Os gráficos de progressão ilustram, de forma diagramática, a relação entre as atividades de leitura e escrita.

\section{Fontes externas}

\subsection{Incorporação de dados externos do Inputlog}

Os softwares Translog-II e CASMACAT somente registram os dados de acionamentos de teclas realizados dentro de uma interface gráfica do usuário. No entanto, em muitos casos, o tradutor utiliza fontes externas, como dicionários eletrônicos, ferramentas de colocação e pesquisas por expressões na internet. Essas atividades não são gravadas no Translog-II ou no CASMACAT, mas o comportamento relativo às pesquisas externas pode ser interessante para fins de investigação e correlação com os dados de atividade do usuário (UAD) do Translog-II.

O Inputlog (LEIJTEN; VAN WAES, 2013) é uma ferramenta para Windows que registra todos os tipos de entrada: acionamentos de teclas e mouse, bem como reconhecimento de fala. Diferentemente do Translog-II e do CASMACAT, o Inputlog não é um aplicativo independente, podendo registrar as atividades do teclado independentemente do aplicativo (do Windows) que está recebendo a entrada de dados. O Inputlog reconhece o aplicativo que está recebendo o foco e, em um arquivo IDFX, armazena essa informação juntamente com os dados da tecla pressionada (ou do clique de mouse) e sua respectiva a marcação de tempo.

Um script ${ }^{14}$, InfuseIDFX.pl, pode ser usado para integrar os dados de registro do Inputlog, IDFX, com os arquivos do Translog-II. Primeiramente, o script "InfuseIDFX.pl" sincroniza os dados de registro do Inputlog e do Translog-II com base nos acionamentos de teclas comuns e, depois, insere, no arquivo de registro do Translog- II, os dados coletados fora da interface gráfica do usuário do Tranlog-II (ou do CASMACAT). Em seguida, os processos de compilação do TPR-DB geram uma tabela EX que indica o uso de fontes externas.

Por exemplo, o Inputlog reconhece a janela que está em foco em um navegador. Os sucessivos acionamentos de teclas e mouse podem ser associados à página web em foco. Dessa forma, pode-se acompanhar e reconstruir as pesquisas na internet.

Por um lado, o Inputlog é universalmente implementável em diferentes aplicativos do Windows; por outro lado, ele não tem como identificar em que parte de um texto o caractere foi digitado. O Inputlog informa quais teclas foram pressionadas, mas não necessariamente

14 O script InfuseIDFX.pl faz parte do TPR-DB e pode ser baixado em: $<$ https://sites.google.com/site/centretranslationinnovation/tpr-db $>$. 
quais caracteres são produzidos ou excluídos, tampouco onde essas operações ocorrem em um texto - a menos que se utilize o Microsoft Word.

Como exemplo, a Tabela 19 exibe um excerto de uma tabela convertida do Inputlog mostrando que o Google Chrome foi usado como fonte externa principal em uma sessão do Translog-II. No instante $33.453 \mathrm{~ms}$, uma aplicação com o nome TASKBAR foi ativada por aproximadamente $0,5 \mathrm{~s}$, seguida por uma busca no Google Chrome, que durou pouco mais do que $32 \mathrm{~s}$. Em seguida, o usuário usou o Menu Iniciar para retornar à janela do Translog-II User, mas ele a deixou novamente após $14.297 \mathrm{~s}$.

Tabela 19 - Uso de fontes externas no TPR-DB

\begin{tabular}{c|c|c|c|c|c|c|c|c|c|c|c|c}
\hline EXid & Study & Session & Focus & Time & Dur & STsegN & STsegL & STidN & STidL & KDidN & KDidL & Edit \\
\hline 0 & N1 & P02_P1 & TASKBAR & 33.453 & 547 & 2 & 1 & 18 & 8 & 27 & 26 & \\
\hline 1 & N1 & P02_P1 & $\begin{array}{c}\text { Google } \\
\text { Chrome }\end{array}$ & 34.000 & 32.813 & 2 & 1 & 18 & 8 & 27 & 26 & EDIT \\
\hline 2 & N1 & P02_P1 & Menu Iniciar & 66.813 & 812 & 2 & 1 & 18 & 8 & 27 & 26 & \\
\hline 3 & N1 & P02_P1 & $\begin{array}{c}\text { Translog-II } \\
\text { User }\end{array}$ & 67.625 & 14.297 & 2 & 1 & 18 & 8 & 27 & 26 & \\
\hline
\end{tabular}

A coluna Edit contém a concatenação das teclas digitadas que ocorreram durante o tempo em foco. Esse valor vai estar vazio caso nenhum acionamento de tecla tenha sido realizado. A seguir, constam três exemplos do que se pode encontrar na cadeia de caracteres representada por EDIT nos $32 \mathrm{~s}$ entre as marcas temporais de $34.000 \mathrm{~ms}$ e $66.818 \mathrm{~ms}$, quando o Google Chrome estava em foco:

1. bring \&\#xD;\&\#xA;

2. emit[..] otional trra[..] adução\&\#xD;\&\#xA;

3. in [.] the $\operatorname{arr}[$.$] ticle\&\#xD;\&\#xA; tradução\&\#xD;\&\#xA;presented\&\#xD;\&\#xA.$

Uma pesquisa geralmente é ativada com o acionamento da tecla "Enter", que nesse caso foi codificado como "\&\#xD;\&\#xA;", e as exclusões estão em colchetes [..]. Sendo assim, na linha (1), o tradutor digitou "bring" e pressionou a tecla "Enter". Na linha (2), o tradutor excluiu dois caracteres duas vezes na cadeia inserida. Pelo arquivo IDFX do Inputlog, não é possível saber quais caracteres foram excluídos, mas é provável que tenham sido, nesta ordem, " $i t$ " e "ra", mediante acionamento da tecla "Backspace", para produzir a sequência de busca "emotional tradução". Na linha (3), foram produzidas três sequências de busca, quais sejam, "in the article", "tradução" e "presented".

Embora possamos reconstruir a pesquisa realizada pelo tradutor na fonte externa, não sabemos quais foram seus resultados. No entanto, é possível rastrear a reação do tradutor 
dentro do Translog-II User. O atributo KDidL indica a última tecla acionada (KDid) antes de o tradutor sair da tela do Translog-II, e o atributo KDidN representa a tecla acionada logo que o tradutor retorna ao programa. De forma parecida, STidL e STidN representam a identificação da palavra-fonte referente a esses acionamentos de teclas, enquanto STsegL e STsegN representam os segmentos-fonte. Sendo assim, a última tecla acionada antes de o tradutor sair do Translog-II User, em 33.453 ms, foi KDidL = 26; e o primeiro toque após seu retorno ao programa foi $\mathrm{KDidN}=27$. Esses dois acionamentos fazem parte da produção para a tradução de $\mathrm{STidL}=8$ e $\mathrm{STidN}=18$, que pertencem aos dois segmentos sucessivos 1 e 2 . Apesar de não ser possível saber o que exatamente o tradutor descobriu ao visitar uma fonte externa, é possível inferir seu efeito ao investigar as ações executadas antes e após a consulta.

\subsection{Adição de colunas às tabelas de resumo do TPR-DB}

Em alguns casos, pode ser desejo do usuário adicionar a algumas tabelas do TPR-DB colunas com anotações próprias. Por exemplo, em um experimento sobre entropia sintática, cada segmento foi anotado manualmente com um conjunto de tríades (em inglês, Triplets), descrevendo a estrutura sintática da sentença. Tais anotações podem ser adicionadas automaticamente a uma tabela de resumo por meio de um script que faz parte do TPR-DB. ${ }^{15}$ Um arquivo com a extensão da tabela especifica o estudo, a sessão e a identificação da unidade, assim como as colunas a serem adicionadas, como mostra a Tabela 20.

Tabela 20 - Anotações com informações suplementares sobre os segmentos em cinco colunas adicionais

\begin{tabular}{c|c|c|c|c|c|c|c}
\hline Study & Session & STseg & SynH & STriplet & TTriplet & PrimeDiff & Prime Prob \\
\hline default & default & default & 0 & - & - & DIFF & 0 \\
\hline BML12 & P03_P1.sg & 2 & 0 & TPI & TPI & PRIME & 1 \\
\hline BML12 & P06_P1.sg & 2 & 0 & TPI & TPI & PRIME & 1 \\
\hline BML12 & P03_P1.sg & 3 & 0.721 & TAI_DAD & TAI_DAD_IAD & DIFF & 0.2 \\
\hline BML12 & P06_P1.sg & 3 & 0,721 & TAI_DAD & TAI_DAD_IAD & DIFF & 0.2 \\
\hline ML12 & P28_P1.sg & 3 & 0,721 & TAI_DAD & TAI_DAD & PRIME & 0.2 \\
\hline BML12 & P32_P1.sg & 3 & 0,721 & TAI_DAD & TAI_DAD & PRIME & 0.2 \\
\hline BML12 & P03_P1.sg & 4 & 0 & TPI_TAD & MPI & DIFF & 0 \\
\hline
\end{tabular}

${ }^{15}$ O script AddExtColumns.pl pode ser baixado em: <https://svn.code.sf.net/p/tprdb/svn/> e invocado com os parâmetros “AddExtColumns.pl -C ExtraColumnsFile-S Study_name”. 


\section{Agradecimentos}

Este trabalho teve apoio do projeto CASMACAT, financiado pela Comissão Europeia (Sétimo Programa-Quadro de Investigação). Somos gratos a todos que contribuíram com o banco de dados e que nos autorizaram o uso de seus dados.

\section{Referências}

ALVES, F.; VALE, D. C. On drafting and revision in translation: a corpus linguistics oriented analysis of translation process data. Translation: Corpora, Computation, Cognition, v. 1, n. 1, p. 105-122, 2011. Disponível em: <http://www.t-c3.org/>. Acesso em: 8 nov. 2017.

CARL, M. Translog-II: A program for recording user activity data for empirical reading and writing research. In: INTERNATIONAL CONFERENCE ON LANGUAGE RESOURCES AND EVALUATION, 8., Istanbul, Tyrkiet, Department of International Language Studies and Computational Linguistics, 21-27 maio 2012a. Proceedings... Istanbul: [s.e.], 2012. p. 26.

CARL, M. The CRITT TPR-DB 1.0: A database for empirical human translation process research. In: O'BRIEN, S.; SIMARD, M.; SPECIA, L. (Ed.). Proceedings of the AMTA 2012 workshop on post-editing technology and practice (WPTP 2012). Stroudsburg, PA: Association for Machine Translation in the Americas (AMTA), 2012b. p. 9-18.

CARL, M.; KAY, M. Gazing and typing activities during translation: a comparative study of translation units of professional and student translators. Meta, v. 56, n. 4, p. 952-975, 2011.

JAKOBSEN, A. L. Translation drafting by professional translators and by translation students. In: HANSEN, G. (Ed.). Empirical translation studies: process and product. Copenhagen: Samfundslitteratur, 2002. p. 191-204.

JAKOBSEN, A. L. Instances of peak performance in translation. Lebende Sprachen, v. 50, n. 3, p. 111-116, 2005.

JAKOBSEN, A. L. Tracking translators' keystrokes and eye movements with Translog. In: ALVSTAD, C.; HILD, A.; TISELIUS, E. (Ed.), Methods and strategies of process research: integrative approaches in translation studies. Amsterdam: John Benjamins, 2011. p. 37-55.

JAKOBSEN, A. L.; SCHOU, L. Translog documentation. In: HANSEN, G. (Ed.). Probing the process in translation methods and results. Copenhagen: Samfundslitteratur, 1999. p. 136.

GERMANN, U. Yawat: Yet Another Word Alignment Tool. In: ACL-08. Proceedings of the 46th Annual Meeting of the Association for Computational Linguistics: HLT demo session. Columbus, OH: Association for Computational Linguistics, 2008. p. 20-23.

LACRUZ, I.; SHREVE, S. Pauses and cognitive effort in post-editing. In post-editing of machine translation: processes and applications. In: O'BRIEN, S.; SIMARD, M.; SPECIA, L.; CARL, M.; BALLING, L. W. (Ed.). Expertise in post-editing: Processes, technology and applications. Cambridge: Scholars Publishing, 2014. p. 246-274.

LEIJTEN, M.; VAN WAES, L. Keystroke logging in writing research: using Inputlog to 
analyze and visualize writing processes. Written Communication, v. 30, n. 3, p. 358-392, 2013.

SANCHIS-TRILLES, G.; ALABAU, V.; BUCK, C.; CARL, M.; CASACUBERTA, F.; GARCÍA-MARTÍNEZ, M.; GERMANN, G.; GONZÁLEZ-RUBIO, J.; HILL, R. L.; KOEHN, P.; LEIVA, L. A.; MESA-LAO, B.; ORTIZ-MARTÍNEZ, D.; SAINT-AMAND, H.; TSOUKALA, C.; VIDAL, E. Interactive translation prediction versus conventional postediting in practice: a study with the CASMACAT workbench. Machine Translation, v. 28, n. 3-4, p. 217-235, 2014.

VANDEPITTE, S.; HARTSUIKER, R. J.; VAN ASSCHE, E. Process and text studies of a translation problem. In: FERREIRA, A.; SCHWIETER, J. W. (Ed.). Psycholinguistic and cognitive inquiries into translation and interpreting. Amsterdã/Filadélfia: John Benjamins, 2015. p. 127-143. 


\section{Apêndice 1}

Ao todo, o TPR-DB contém mais de 580 horas de produção textual considerando o atributo Fdur. Contribuíram para as 1689 sessões 132 tradutores diferentes, que produziram um total de mais de 660.000 palavras em nove línguas diferentes.

$\mathrm{O}$ par linguístico en $\rightarrow$ es (inglês-espanhol) é de longe aquele que conta com maior representatividade no TPR-DB, com 660 sessões, 500.000 palavras nas traduções e mais de 320 horas de produção considerando o atributo Fdur. O segundo par linguístico mais bem representado é o en $\rightarrow$ hi (inglês-hindu), com 161 sessões, mais de 20.000 palavras nas traduções e mais de 46 horas de produção pelo atributo Fdur. O terceiro par linguístico é o en $\rightarrow$ de (inglês-alemão), com 146 sessões, mais de 24.000 itens (tokens) nas traduções e mais de 24 horas de produção pelo atributo Fdur. Em seguida, vem o par en $\rightarrow$ da (inglêsdinamarquês), com 127 sessões, mais de 18.000 itens nas traduções e 12 horas de produção segundo o atributo Fdur. Os outros pares linguísticos do TPR-DB envolvem mais de 20 direções de tradução a partir da combinação de sete línguas-fonte diferentes com 16 línguasalvo distintas (inclusive alguns pares linguísticos que não aparecem na Tabela 21). Remete-se o leitor à página do TPR-DB para uma versão atualizada do conteúdo do banco de dados.

Cada estudo no TPR-DB foi conduzido a partir de uma ou mais perguntas de pesquisa. Os estudos podem ser resumidamente agrupados da seguinte forma:

(A) O TPR-DB contém dez estudos conduzidos com as três diferentes interfaces do CASMACAT:

1. ALG14: compara tradutores profissionais e falantes bilíngues durante uma pós-edição com o terceiro protótipo do CASMACAT, apresentando visualizações dos alinhamentos entre as palavras;

2. CEMPT13: contém gravações de pós-edições com o segundo protótipo do CASMACAT, apresentando a tradução automática interativa;

3. CFT12: contém dados do primeiro teste de campo do CASMACAT em junho de 2012, comparando a pós-edição com a tradução do zero;

4. CFT13: contém dados do segundo teste de campo do CASMACAT em junho de 2013, comparando a pós-edição com a tradução automática interativa;

5. CFT14: contém dados do segundo teste de campo do CASMACAT em junho de 2014, comparando a tradução automática interativa com o aprendizado online;

6. EFT14: compara a aprendizagem ativa e a aprendizagem online para 
previsões em traduções interativas;

7. JN13: foi gravado com o segundo protótipo do CASMACAT e apresenta o recurso de alinhamentos de palavras e tradução automática interativa;

8. LS14: investiga, com o terceiro protótipo do CASMACAT, os efeitos do aprendizado com a pós-edição interativa durante um período de seis semanas (estudo longitudinal);

9. PFT13: pré-teste que antecede o segundo teste de campo do CASMACAT;

10. PFT14: pré-teste que antecede o terceiro teste de campo do CASMACAT. 
Tabela 21 - Tabela de resumo dos estudos do TPR-DB: continuação abaixo

\begin{tabular}{|c|c|c|c|c|c|c|c|c|c|c|c|}
\hline Study & Sess & SL & $\mathrm{TL}$ & Task & Texts & Part & Fdur & Kdur & Pdur & Stok & Ttok \\
\hline ACS08 & 30 & en & $\mathrm{da}$ & $\mathrm{T}$ & 4 & 17 & 4.6776 & 2.9704 & 1.9332 & 5085 & 5075 \\
\hline ACS08 & 30 & en & en & $\mathrm{C}$ & 4 & 17 & 2.0436 & 1.8316 & 1.6013 & 5099 & 5109 \\
\hline ALG14 & 8 & en & es & $\mathrm{P}$ & 2 & 8 & 2.6018 & 0.4854 & 0.1747 & 4460 & 4807 \\
\hline ALG14 & 8 & en & es & PA & 2 & 8 & 2.7954 & 0.4437 & 0.1692 & 4460 & 4801 \\
\hline BD08 & 10 & en & $\mathrm{da}$ & $\mathrm{T}$ & 1 & 10 & 1.4575 & 0.7493 & 0.448 & 1100 & 1056 \\
\hline BD13 & 8 & en & $\mathrm{da}$ & $\mathrm{T}$ & 2 & 8 & 0.8079 & 0.5368 & 0.3213 & 786 & 751 \\
\hline BD13 & 10 & en & $\mathrm{da}$ & $\mathrm{P}$ & 2 & 10 & 0.4412 & 0.1074 & 0.0569 & 970 & 1014 \\
\hline$B M L 12$ & 64 & en & es & $\mathrm{P}$ & 6 & 32 & 4.6394 & 0.9079 & 0.4418 & 9012 & 10,216 \\
\hline BML12 & 63 & en & es & $\mathrm{T}$ & 6 & 32 & 9.8032 & 5.9308 & 3.8062 & 8936 & 10,102 \\
\hline$B M L 12$ & 60 & en & es & $\mathrm{E}$ & 6 & 30 & 3.7009 & 0.9657 & 0.4729 & 8468 & 9594 \\
\hline CEMPT13 & 20 & en & $\mathrm{pt}$ & PIA & 2 & 20 & 6.634 & 1.823 & 0.5387 & 6706 & 6840 \\
\hline CEMPT13 & 20 & en & $\mathrm{pt}$ & $\mathrm{P}$ & 2 & 20 & 5.5943 & 1.2678 & 0.5732 & 6494 & 6585 \\
\hline CFT13 & 27 & en & es & $\mathrm{R}$ & 26 & 4 & 8.3388 & 0.9733 & 0.4413 & 26,919 & 28,738 \\
\hline CFT13 & 27 & en & es & PI & 9 & 9 & 30.0923 & 10.2351 & 3.3044 & 31,752 & 33,871 \\
\hline CFT13 & 27 & en & es & $\mathrm{P}$ & 9 & 9 & 28.167 & 8.0677 & 03.51 & 31,294 & 33,770 \\
\hline CFT13 & 27 & en & es & PIA & 9 & 9 & 35.5658 & 11.2626 & 3.9125 & 31,838 & 34,047 \\
\hline CFT14 & 7 & en & es & $\mathrm{RE}$ & 7 & 3 & 3.8435 & 0.2465 & 0.0586 & 20,341 & 22,015 \\
\hline CFT14 & 7 & en & es & $\mathrm{R}$ & 7 & 4 & 3.2497 & 0.3687 & 0.1485 & 20,273 & 22,251 \\
\hline CFT14 & 7 & en & es & $\mathrm{P}$ & 2 & 7 & 16.8321 & 7.9316 & 3.418 & 20,273 & 22,067 \\
\hline CFT14 & 7 & en & es & PEI & 2 & 7 & 15.8297 & 8.1574 & 3.4917 & 20,341 & 22,284 \\
\hline DG01 & 60 & fr & $\mathrm{pl}$ & $\mathrm{T}$ & 2 & 60 & 33.8564 & 17.5784 & 11.2075 & 25,380 & 20,329 \\
\hline EFT14 & 11 & en & es & PIVO & 3 & 11 & 10.221 & 5.2041 & 2.2521 & 12,437 & 13,549 \\
\hline EFT14 & 11 & en & es & PI & 3 & 11 & 11.9495 & 6.8647 & 3.2755 & 12,437 & 13,696 \\
\hline EFT14 & 10 & en & es & PIVA & 3 & 10 & 10.7885 & 5.1993 & 2.3594 & 11,327 & 12,472 \\
\hline GS12 & 8 & es & en & $\mathrm{P}$ & 4 & 4 & 4 & 4 & 2.1901 & 0.3586 & 0.1909 \\
\hline HLR13 & 15 & en & et & $\mathrm{T}$ & 3 & 5 & 2.5457 & 1.1214 & 0.673 & 1535 & 1186 \\
\hline JIN15 & 18 & en & $\mathrm{zh}$ & S & 1 & 18 & 2.0227 & 0.2641 & 0.0455 & 1947 & 1728 \\
\hline
\end{tabular}

(continua) 
Tabela 21 - (continuação)

\begin{tabular}{|c|c|c|c|c|c|c|c|c|c|c|c|}
\hline Study & Sess & SL & $\mathrm{TL}$ & Task & Texts & $\mathrm{Pa}$ & Fdur & Kdur & Pdur & Stok & Ttok \\
\hline JIN15 & 18 & en & $\mathrm{zh}$ & $\mathrm{P}$ & 1 & 18 & 4.5318 & 0.8192 & 0.1442 & 1998 & 1845 \\
\hline JIN15 & 17 & en & $\mathrm{zh}$ & $\mathrm{R}$ & 1 & 17 & 2.594 & 0.3451 & 0.0567 & 1946 & 1833 \\
\hline JLG10 & 10 & en & $\mathrm{pt}$ & $\mathrm{T}$ & 3 & 5 & 5.6048 & 2.1218 & 1.2302 & 2577 & 2781 \\
\hline JLG10 & 10 & $\mathrm{pt}$ & en & $\mathrm{T}$ & 3 & 5 & 5.6391 & 2.0787 & 1.1718 & 2611 & 2621 \\
\hline JN13 & 4 & en & de & PIA & 2 & 4 & 2.7428 & 0.7284 & 0.2735 & 2590 & 2668 \\
\hline JN13 & 4 & en & de & $\mathrm{P}$ & 2 & 4 & 2.3311 & 0.6374 & 0.2189 & 2590 & 2571 \\
\hline КТНJ08 & 69 & en & $\mathrm{da}$ & $\mathrm{T}$ & 3 & 24 & 7.4469 & 5.6824 & 3.8183 & 10,571 & 10,667 \\
\hline LS14 & 60 & en & es & PI & 24 & 5 & 53.376 & 22.0971 & 9.5166 & 72,109 & 80,278 \\
\hline LS14 & 60 & en & es & $\mathrm{P}$ & 24 & 5 & 51.725 & 17.3211 & 7.4178 & 72,126 & 80,454 \\
\hline LWB09 & 40 & $\mathrm{da}$ & en & $\mathrm{T}$ & 3 & 18 & 3.7061 & 2.8926 & 2.0511 & 5652 & 6206 \\
\hline MS12 & 19 & en & $\mathrm{zh}$ & $\mathrm{P}$ & 6 & 11 & 2.6953 & 0.4817 & 0.0497 & 2708 & 2562 \\
\hline MS12 & 15 & en & $\mathrm{zh}$ & $\mathrm{T}$ & 5 & 10 & 3.7369 & 1.0512 & 0.1088 & 2061 & 1916 \\
\hline MS12 & 10 & en & $\mathrm{zh}$ & $\mathrm{E}$ & 5 & 8 & 0.7714 & 0.1564 & 0.0183 & 1295 & 1203 \\
\hline MS13 & 16 & $\mathrm{zh}$ & $\mathrm{pt}$ & $\mathrm{P}$ & 2 & 16 & 2.7139 & 0.9211 & 0.4443 & 1410 & 1648 \\
\hline MS13 & 16 & $\mathrm{pt}$ & $\mathrm{zh}$ & $\mathrm{T}$ & 2 & 16 & 2.3327 & 0.7687 & 0.1161 & 1386 & 1378 \\
\hline MS13 & 22 & $\mathrm{zh}$ & $\mathrm{pt}$ & $\mathrm{T}$ & 2 & 22 & 4.1631 & 2.1803 & 1.2265 & 1938 & 2216 \\
\hline MS13 & 18 & $\mathrm{pt}$ & $\mathrm{zh}$ & $\mathrm{P}$ & 2 & 18 & 2.555 & 0.6698 & 0.0934 & 1555 & 1507 \\
\hline NJ12 & 39 & en & hi & $\mathrm{T}$ & 6 & 20 & $14.469^{\prime}$ & 7.5368 & 3.3156 & 5505 & 5784 \\
\hline NJ12 & 61 & en & hi & $\mathrm{P}$ & 6 & 20 & 17.440 & 6.8654 & 3.0615 & 8581 & 9365 \\
\hline PFT13 & 9 & en & es & $\mathrm{P}$ & 1 & 9 & 2.0861 & 0.3154 & 0.1406 & 3035 & 3144 \\
\hline PFT13 & 19 & en & es & PI & 1 & 15 & 5.2058 & 1.5351 & 0.4267 & 6689 & 7437 \\
\hline PFT13 & 16 & en & es & PIC & 3 & 16 & 2.7853 & 0.744 & 0.1518 & 5396 & 5147 \\
\hline PFT13 & 15 & en & es & PEI & 3 & 15 & 2.4784 & 0.4741 & 0.0669 & 4611 & 4666 \\
\hline PFT13 & 16 & en & es & PIL & 3 & 16 & 2.7226 & 0.6761 & 0.1511 & 5572 & 5344 \\
\hline PFT14 & 3 & en & es & PIVO & 2 & 3 & 2.1558 & 0.6775 & 0.1622 & 3245 & 3150 \\
\hline PFT14 & 2 & en & es & PIVA & 1 & 2 & 2.0228 & 0.7255 & 0.1843 & 2286 & 2184 \\
\hline PFT14 & 2 & en & es & PIV & 2 & 2 & 1.987 & 0.7667 & 0.1905 & 2161 & 2077 \\
\hline RH12 & 2 & es & es & A & 2 & 2 & 2.9849 & 0.9786 & 0.6398 & 1207 & 1207 \\
\hline ROBOT14 & 40 & in & $\mathrm{nl}$ & $\mathrm{P}$ & 8 & 10 & 10.870 & 3.2467 & 1.5417 & 7375 & 7527 \\
\hline ROBOT14 & 40 & en & $\mathrm{nl}$ & $\mathrm{T}$ & 8 & 10 & 12.2457 & 5.1006 & 3.1753 & 7375 & 7329 \\
\hline$S G 12$ & 46 & en & de & E & 6 & 23 & 7.0716 & 1.8571 & 0.9342 & 6522 & 6741 \\
\hline$S G 12$ & 45 & en & de & $\mathrm{P}$ & 6 & 23 & 8.027 & 1.9976 & 1.055 & 6352 & 6470 \\
\hline$S G 12$ & 47 & en & de & $\mathrm{T}$ & 6 & 24 & 11.7259 & 4.7344 & 2.9421 & 6632 & 6777 \\
\hline$T D A 14$ & 48 & en & en & $\mathrm{C}$ & 6 & 8 & 3.8335 & 3.5653 & 2.6617 & 6792 & 6779 \\
\hline WARDHA13 & 34 & en & hi & $\mathrm{T}$ & 6 & 18 & 15.2298 & 3.6917 & 0.5553 & 4832 & 4790 \\
\hline WARDHA13 & 31 & hi & hi & $\mathrm{C}$ & 6 & 18 & 11.49 & 5.3097 & 0.7569 & 4365 & 4104 \\
\hline WARDHA13 & 27 & en & hi & $\mathrm{P}$ & 6 & 15 & 8.0582 & 1.9611 & 0.4418 & 3780 & 4016 \\
\hline ZHРT12 & 12 & $\mathrm{zh}$ & $\mathrm{pt}$ & $\mathrm{T}$ & 1 & 12 & 3.5244 & 1.4856 & 0.851 & 1104 & 1603 \\
\hline Total & 1689 & 7 & 9 & 15 & 132 & 418 & 586.769 & 217.2386 & 100.2227 & 702,701 & 660,595 \\
\hline
\end{tabular}

A tabela mostra informações do resumo do TPR-DB para cada sessão: tarefa, direcionalidade e par linguístico, número de textos distintos, número de participantes, duração da produção (Fdur, Kdur e Pdur), assim como o 
comprimento total, em palavras (itens, ou tokens), do texto-fonte (STok) e do texto-alvo (TTok).

(B) O objetivo do experimento multilinguístico é comparar a tradução a partir do zero $(\mathrm{T})$, a pós-edição bilíngue $(\mathrm{P})$ e a edição, ou pós-edição monolíngue $(\mathrm{E})$, entre diferentes tradutores e línguas. Os seis textos-fonte em inglês foram traduzidos por tradutores em formação e tradutores experientes. Três (1-3) são textos jornalísticos, e três (4-5) são textos de sociologia extraídos de uma enciclopédia. Os textos foram sistematicamente alternados de forma que todos eles fossem traduzidos por todos os tradutores e cada tradutor traduzisse dois textos diferentes em cada uma das modalidades. Os estudos desse grupo são:

11. BML12: contém dados de tradução, pós-edição e edição dos seis textos do inglês para o espanhol;

12. KTHJ08: contém apenas dados de tradução dos textos jornalísticos (1-3);

13. MS12: contém dados de tradução, pós-edição e edição dos seis textos do inglês para o chinês;

14. NJ12: contém dados de tradução, pós-edição e edição dos seis textos do inglês para o hindu que foram realizadas por tradutores profissionais;

15. SG12: contém dados de tradução, pós-edição e edição dos seis textos do inglês para o alemão;

16. TDA14: contém dados de participantes que fizeram cópias dos seis textos em inglês;

17. WARDHA13: contém dados de tradução, pós-edição e edição dos seis textos do inglês para o hindu que foram realizadas por estudantes.

(C) O BD-TTP contém, ainda, alguns experimentos individuais que foram conduzidos com o Translog-II. São eles:

18. ACS08: explora, por meio da análise dos tempos do olhar associados às expressões, a forma como os tradutores processam o significado de expressões não literais;

19. BD08: envolve tradutores profissionais dinamarqueses trabalhando com o par linguístico en $\rightarrow$ da;

20. BD13: envolve estudantes do Ensino Médio realizando tarefas de tradução e pós-edição do inglês para o dinamarquês; 
21. DG01: compara estudantes, tradutores profissionais e tradutores não profissionais em termos de representação do texto;

22. GS12: contém dados de pós-edição de quatro notícias traduzidas do espanhol para o inglês;

23. HLR13: investiga as traduções de três textos distintos do inglês para o estoniano realizadas por cinco participantes;

24. JLG10: investiga traduções direta (L2-L1) e inversa (L1-L2) no par linguístico inglês-português (do Brasil);

25. LWB09: traz os resultados de um experimento com rastreamento ocular em que tradutores profissionais traduziram dois textos do dinamarquês (L1) para o inglês (L2);

26. MS13: investiga o comportamento do tradutor durante a tradução e a pósedição no par linguístico português-chinês (em ambas as direções);

27. RH12: investiga a produção autoral de notícias por dois jornalistas espanhóis;

28. ROBOT14: investiga o uso de fontes externas durante a tradução e a pósedição;

29. ZHPT12: investiga o comportamento do tradutor ao traduzir textos jornalísticos, tendo como foco o processo tradutório durante o processamento de expressões não literais (metafóricas). 


\section{Apêndice 2}

Durante cada sessão, é conduzida uma tarefa (em inglês, Task) específica, que pode ser:

- A: escrita autoral de um texto jornalístico, com a língua-fonte e a língua-alvo sendo as mesmas;

- C: cópia (manual) de um texto da janela-fonte para a janela-alvo, com a línguafonte e a língua-alvo sendo as mesmas;

- E: edição de uma tradução automática sem acesso ao texto-fonte (pós-edição monolíngue);

- P: pós-edição de uma tradução automática (com acesso ao texto-fonte e sem consultas externas);

- R: revisão de um texto pós-editado;

- T: tradução "do zero".

No contexto do CASMACAT, diversas situações de pós-edição foram investigadas, a saber:

- PA: pós-edição tradicional com acesso, ativado por mouse ou cursor, aos alinhamentos entre texto-fonte (ST) e texto-alvo (TT);

- PI: pós-edição avançada com o aporte de previsões interativas de tradução (do inglês, interactive translation prediction, ou ITP) / tradução automática interativa;

- PIA: pós-edição avançada com o aporte de ITP e com acesso aos alinhamentos ST-TT (opção de visualização);

- PIC: pós-edição avançada com o aporte de ITP e com acesso aos alinhamentos ST-TT (opção de visualização);

- PIO: pós-edição avançada com o aporte de ITP e de técnicas de aprendizagem online;

- PIL: pós-edição avançada com o aporte de ITP e com acesso ao texto pós-editado (sufixo) em cinza (opção de visualização);

- PIV: pós-edição avançada com o aporte de ITP e com acesso a uma barra de busca e substituição, a alinhamentos e a opções de ITP ativadas pelo mouse;

- PIVA: pós-edição avançada com o aporte de ITP e de técnicas de aprendizagem ativa;

- PIVO: pós-edição avançada com o aporte de ITP e de técnicas de aprendizagem 
online.

Revista Graphos, Edição Especial, 2018 | UFPB/PPGL | ISSN 1516-1536 


\section{Apêndice 3}

Este apêndice lista todos os atributos utilizados no TPR-DB 2.0 em suas tabelas de unidades. Há, no total, 275 atributos, dos quais 111 são distintos, nos onze tipos de tabelas discutidos neste artigo. Esses atributos foram agrupados em doze tipos, de acordo com o que descrevem (sessão, segmento, item, acionamento de tecla, comportamento ocular etc.). Entre parênteses estão indicadas as tabelas de unidades em que os atributos aparecem.

1. Dados de sessão (informações relativas às sessões de um estudo):

- Study: nome do estudo como consta no TPR-DB (AU, EX, PU, SG, SS, ST, TT);

- Session: nome da sessão, um composto formado a partir da identificação dada ao participante, ao texto e à tarefa (AU, CU, EX, PU, SG, SS, ST, TT);

- Text: identificação do texto utilizado no estudo (AU, SS, ST, TT);

- Task: tipo de tarefa, cf. Apêndice 2 (AU, SS, ST, TT);

- Part: identificação do participante do estudo (AU, ST, TT, SS);

- SL: língua do texto-fonte (AU, SS, ST, TT);

- TL: língua do texto-alvo (AU, SS, ST, TT);

- Break: duração do intervalo da sessão (SS);

- TimeR: tempo de início da fase de revisão (SS);

- TimeD: tempo de início da fase de redação (SS).

2. Dados do segmento (informações relativas aos segmentos):

- Seg: identificação do segmento-fonte ou do segmento-alvo, dependendo do atributo Win, ou seja, da janela (FD);

- STseg: identificação do segmento-fonte (AU, PU, SG, SS, ST);

- Nedit: número de vezes que o segmento foi editado (SG);

- TTseg: identificação do segmento-alvo (AU, CU, KD, PU, TT, SG, SS);

- LenS: comprimento, em caracteres, do segmento-fonte (SG, SS);

- LenT: comprimento, em caracteres, do segmento-alvo (SG, SS);

- LenMT: comprimento, em caracteres, do segmento traduzido automaticamente $(\mathrm{SG})$;

- TokS: número de itens-fonte (tokens) no segmento (SG, SS);

- TokT: número de itens-alvo (tokens) no segmento (SG, SS);

- Literal: grau de literalidade de segmento (SG). 
3. Itens (informações relativas aos itens, ou palavras, do texto-fonte e do texto-alvo):

- STId: identificação única do item (token) do texto-fonte (FD, KD, PU, ST, TT);

- TTId: identificação única do item (token) do texto-alvo (FD, KD, PU, ST, TT);

- SAU: cadeia de caracteres do texto-fonte (AU);

- TAU: cadeia de caracteres do texto-alvo (AU);

- SAUnbr: número de itens (tokens) do lado-fonte da unidade de alinhamento (AU, ST, TT);

- TAUnbr: número de itens (tokens) do lado-alvo da unidade de alinhamento (AU, ST, TT);

- SToken: item (token) do texto-fonte (ST, TT);

- TToken: item (token) do texto-alvo (ST, TT);

- Lemma: lema do item (ST, TT);

- PoS: classe gramatical do item (ST, TT);

- PosS: classe gramatical da sequência do item-fonte (PU);

- PosT: classe gramatical da sequência do item-alvo (PU);

- Prob1: probabilidade de ocorrência do monograma (ST, TT);

- Prob2: probabilidade de ocorrência do bigrama (ST, TT).

4. Métrica de literalidade tradutória:

- AltT: número de diferentes alternativas de tradução (ST);

- CountT: número de escolhas de tradução atuais observadas (ST);

- ProbT: probabilidade de escolhas de tradução atuais (ST);

- HTra: entropia de tradução da palavra (SG, ST);

- HSeg: entropia de segmentação da tradução (SG, ST);

- Cross: valor Cross do item (AU, ST, TT);

- CrossS: valor Cross para item-fonte (PU, SG);

- CrossT: valor Cross para item-alvo (PU, SG);

- Literal: grau de literalidade de segmento (SG).

5. Acionamentos de teclas (informações relativas às atividades no teclado):

- KDid: identificação do acionamento de tecla (KD);

- Del: número de exclusões manuais e automáticas (AU, PU, ST, TT);

- Ins: número de inserções manuais e automáticas (AU, PU, ST, TT);

- Adel: número de exclusões realizadas automaticamente (SG, SS); 
- Ains: número de inserções realizadas automaticamente (SG, SS);

- Mdel: número de exclusões realizadas manualmente (SG, SS);

- Mins: número de inserções realizadas manualmente (SG, SS);

- Char: caractere (UTF8) inserido ou excluído (KD);

- Munit: número de microunidades (AU, ST, TT);

- Edit: sequência de acionamentos de teclas na produção da cadeia no TT (AU, EX, FD, PU, ST, TT);

- Edit1: sequência de acionamentos de teclas da primeira microunidade (AU, ST, TT);

- Edit2: sequência de acionamentos de teclas da segunda microunidade (AU, $\mathrm{ST}, \mathrm{TT})$;

- InEff: medida de ineficiência para a geração do segmento (AU, ST, TT);

- Scatter: quantidade de produção textual não linear (PU, SG, SS).

6. Olhar sobre a janela-fonte e sobre a janela-alvo:

- Path: sequência de fixações na janela do texto-fonte ou do texto-alvo (FU);

- FFTime: marcação temporal do início da primeira fixação (ST, TT);

- FFDur: duração da primeira fixação (ST, TT);

- FPDurS: duração da primeira passagem na unidade do texto-fonte (AU, ST, TT);

- FPDurT: duração da primeira passagem na unidade do texto-alvo (AU, ST, TT);

- FixS: número de fixações na unidade do texto-fonte (AU, PU, SG, SS, ST, TT);

- FixT: número de fixações na unidade do texto-alvo (AU, PU, SG, SS, ST, TT);

- TrtS: tempo total de olhar (gaze) na unidade do texto-fonte (AU, SG, SS, $\mathrm{ST}, \mathrm{TT})$;

- TrtT: tempo total de olhar (gaze) na unidade do texto-alvo (AU, SG, SS, $\mathrm{ST}, \mathrm{TT})$;

- FixS1: número de fixações na unidade do texto-fonte durante a produção da primeira microunidade (AU, ST, TT);

- FixS2: número de fixações na unidade do texto-fonte durante a produção da segunda microunidade (AU, ST, TT);

- FixT1: número de fixações na unidade do texto-alvo durante a produção da 
primeira microunidade (AU, ST, TT);

- FixT2: número de fixações na unidade do texto-alvo durante a produção da segunda microunidade (AU, ST, TT);

- RPDur: duração do percurso de regressão (ST, TT);

- Regr: valor booleano que indica se uma regressão partiu de um item (ST, TT).

7. Atividades concomitantes de olhar e acionamento de teclas:

- ParalK: atividade de teclado paralela à atividade ocular (FU, FD);

- ParalS: atividade ocular no texto-fonte paralela à digitação (PU);

- ParalT: atividade ocular no texto-alvo paralela à digitação (PU);

- ParalS1: atividade ocular no texto-fonte paralela à digitação da primeira microunidade (AU, ST, TT);

- ParalS2: atividade ocular no texto-fonte paralela à digitação da segunda microunidade (AU, ST, TT);

- ParalT1: atividade ocular no texto-alvo paralela à digitação da primeira microunidade (AU, ST, TT);

- ParalT2: atividade ocular no texto-alvo paralela à digitação da segunda microunidade (AU, ST, TT).

8. Marcações temporais de início e duração de unidades e fases:

- Dur: duração da produção da unidade (AU, CU, EX, FD, FU, PU, SG, SS, ST, TT);

- Dur1: duração da produção da primeira microunidade (AU, ST, TT);

- Dur2: duração da produção da segunda microunidade (AU, ST, TT);

- Fdur: duração da produção do segmento excluindo pausas de digitação iguais ou superiores a $200 \mathrm{~s}(\mathrm{SG}, \mathrm{SS})$;

- Kdur: duração de atividade contínua de teclado excluindo pausas de digitação iguais ou superiores a $5 \mathrm{~s}(\mathrm{SG}, \mathrm{SS})$;

- Pdur: duração de atividade contínua de teclado excluindo pausas de digitação iguais ou superiores a $1 \mathrm{~s}(\mathrm{SG}, \mathrm{SS})$;

- Pnum: número de unidades de produção (SG, SS);

- Time: marcação temporal de início da unidade (CU, EX, FD, FU, KD, PU);

- Time1: marcação temporal de início da primeira microunidade (AU, ST, TT);

- Time2: marcação temporal de início da segunda microunidade (AU, ST, TT);

- TimeR: tempo de início da fase de revisão (SS); 
- TimeD: tempo de início da fase de redação (SS).

9. Pausa anterior ao início de uma unidade:

- Pause: pausa entre o fim da unidade anterior e o início da unidade atual (FU, PU);

- Pause1: pausa entre o fim da unidade anterior e o início da primeira microunidade (AU, ST, TT);

- Pause2: pausa entre o fim da unidade anterior e o início da segunda microunidade (AU, ST, TT).

10. Informações relativas à interface gráfica do usuário (GUI):

- Win: janela em que foi gravada a atividade ocular, sendo " 1 " para texto-fonte e "2" para texto-alvo (FD);

- Cursor: posição do caractere em que se registraram a atividade, os acionamentos de teclas e as fixações (FD, KD).

\section{Fontes externas:}

- Focus: nome da janela em primeiro plano (EX);

- KDidL: identificação do último acionamento de tecla anterior à saída da tela do Translog-II (EX);

- KDidN: identificação do primeiro acionamento de tecla posterior ao retorno à tela do Translog-II (EX);

- STidN: identificação do primeiro item (token) do texto-fonte posterior ao retorno à tela do Translog-II $(\mathrm{EX})$;

- STidL: identificação do último item (token) do texto-fonte anterior à saída da tela do Translog-II (EX);

- STsegL: identificação do segmento (do texto-fonte) do último evento (EX);

- STsegN: identificação do segmento (do texto-fonte) do evento subsequente (EX).

12. Outros atributos:

- Type: tipo de acionamento de tecla: $[\mathrm{AM}] \mathrm{ins},[\mathrm{AM}] \mathrm{del}(\mathrm{KD})$;

- Type: tipo de unidade de atividade, como explicado na Seção 4.5 (CU);

- Label: rótulo das unidades de atividade

$(\mathrm{CU})$. 